\title{
The Reversible-Irreversible Approach to Schiff Base Macrocycles: Access to Isomeric Macrocycles With Multiple Salphen Pockets
}

Peter D. Frischmann, Jian Jiang, Joseph K.-H. Hui, Joseph J. Grzybowski, and Mark J. MacLachlan*

Department of Chemistry, University of British Columbia, 2036 Main Mall, Vancouver, BC V6T 1Z1 CANADA

mmaclach@chem.ubc.ca

Table of contents:

Materials

Equipment

Experimental

Crystallography

Kinetics

Figure S1

Figure S2

Figure S3

Figure S4

Figure S5

Figure S6

Figure S7

Figure S8

Figure S9

Figure S10

Figure S11

Figure S12

Figure S13

Figure S14

Figure S15

Figure S16

Figure S17

Figure S18

Figure S19

Figure S20

Figure S21

Figure S22
Exchange Kinetics (M vs. t)

${ }^{1} \mathrm{H}$ NMR spectrum of $\mathbf{1}$

${ }^{13} \mathrm{C}$ NMR spectrum of $\mathbf{1}$

${ }^{1} \mathrm{H}$ NMR spectrum of $\mathbf{2}$

${ }^{13} \mathrm{C}$ NMR spectrum of 2

${ }^{1} \mathrm{H}$ NMR spectrum of $\mathbf{3}$

${ }^{13} \mathrm{C}$ NMR spectrum of 3

${ }^{1} \mathrm{H}$ NMR spectrum of $\mathbf{4}$

${ }^{13} \mathrm{C}$ NMR spectrum of 4

${ }^{1} \mathrm{H}$ NMR spectrum of 5

${ }^{13} \mathrm{C}$ NMR spectrum of 5

${ }^{1} \mathrm{H}$ NMR spectrum of $\mathbf{5 b}$

${ }^{13} \mathrm{C}$ NMR spectrum of $5 \mathbf{b}$

${ }^{1} \mathrm{H}$ NMR spectrum of $\mathbf{6}$

${ }^{13} \mathrm{C}$ NMR spectrum of 6

${ }^{1} \mathrm{H}$ NMR spectrum of 8

${ }^{13} \mathrm{C}$ NMR spectrum of 8

${ }^{1} \mathrm{H}$ NMR spectrum of $\mathbf{1 0}$

${ }^{13} \mathrm{C}$ NMR spectrum of $\mathbf{1 0}$

${ }^{1} \mathrm{H}$ NMR spectrum of 11a

${ }^{13} \mathrm{C}$ NMR spectrum of 11a

${ }^{1} \mathrm{H}$ NMR spectrum of $\mathbf{1 1 b}$
Page S3

Page S3

Page S4

Page S9

Page S9

Page S11

Page S11

Page S12

Page S12

Page S13

Page S13

Page S14

Page S14

Page S15

Page S15

Page S16

Page S16

Page S17

Page S17

Page S17

Page S18

Page S18

Page S19

Page S19

Page S19

Page S20

Page S20 
Figure S23 ${ }^{13} \mathrm{C}$ NMR spectrum of 11b

Figure S24 ${ }^{1} \mathrm{H}$ NMR spectrum of 12

Figure S25 ${ }^{13} \mathrm{C}$ NMR spectrum of 12

Figure S26 ESI mass spectrum of $\mathbf{1 2}$

Figure S27 $\quad{ }^{1} \mathrm{H}$ NMR spectrum of $\mathbf{1 3}$

Figure S28 ${ }^{13} \mathrm{C}$ NMR spectrum of $\mathbf{1 3}$

Figure S29 MALDI-TOF mass spectrum of 13

Figure S30

Figure S31

Figure S32

Figure S33
${ }^{1} \mathrm{H}$ NMR spectrum of $\mathbf{1 4}$

${ }^{13} \mathrm{C}$ NMR spectrum of 14

MALDI-TOF MS of 14

MALDI-TOF MS of 14 filtrate
Page S21

Page S21

Page S21

Page S22

Page S22

Page S23

Page S23

Page S24

Page S24

Page S25

Page S26 
Materials: The synthesis of $N$-salicylidene- $p$-anisidine, $\mathbf{1},{ }^{1}$ has been previously reported but in this Letter it has been synthesized by a different route and extensively characterized so we include the synthesis and characterization. Compounds $5,5 \mathbf{b}$, and $\mathbf{6}$ were synthesized by literature procedures, ${ }^{2}$ but their complete characterization is included here. Compounds 7, ${ }^{3}$ 9a-b, ${ }^{4}$ 2-benzoyl-4-methylphenol, ${ }^{5}$ 3,6-diformylcatechol, ${ }^{6}$ and 4,6-diformylresorcinol ${ }^{7}$ were prepared by literature methods. Deuterated solvents were purchased from Cambridge Isotope Laboratories.

Equipment: All reactions were carried out under air unless otherwise noted. ${ }^{1} \mathrm{H}$ and ${ }^{13} \mathrm{C}$ NMR spectra were recorded on either a Bruker AV-300 or AV-400 spectrometer. ${ }^{13} \mathrm{C}$ NMR spectra were recorded using a proton decoupled pulse sequence. ${ }^{1} \mathrm{H}$ and ${ }^{13} \mathrm{C}$ NMR spectra were calibrated to the residual protonated solvent at $\delta 7.27$ and $\delta 77.23$, respectively, in $\mathrm{CDCl}_{3}$ or at $\delta 5.32$ and $\delta 54.00$, respectively, in $\mathrm{CD}_{2} \mathrm{Cl}_{2}$. UV-Vis spectra were obtained in $\mathrm{CH}_{2} \mathrm{Cl}_{2}$ or $\mathrm{MeOH}$ (ca. $1 \times 10^{-6} \mathrm{~mol} \mathrm{~L}^{-1}$ ) on a Varian Cary $5000 \mathrm{UV}$-Visnear-IR spectrophotometer using a $1 \mathrm{~cm}$ quartz cuvette. IR spectra were collected neat in the solid state on a Thermo Nicolet 6700 FT-IR spectrometer or in a $\mathrm{KBr}$ pellet on a Nicolet 4700 FT-IR spectrometer. All mass spectrometry experiments were conducted at the UBC Microanalytical Services Laboratory. EI-MS was conducted on a Kratos MS-50 spectrometer. MALDI-TOF mass spectrometry was performed on a Bruker Biflex IV instrument with a dithranol matrix. Electrospray ionization (ESI) mass spectra were obtained on a Bruker Esquire LC instrument in $\mathrm{MeOH}$ (ca. 1 × $10^{-4} \mathrm{~mol} \mathrm{~L}^{-1}$ ). Elemental analyses $(\mathrm{C}, \mathrm{H}, \& \mathrm{~N})$ were performed at the UBC Microanalytical Services Laboratory. Melting points were obtained on a Fisher-John's melting point apparatus. 


\section{Experimental:}

Scheme 1. Synthesis of Aldimines and Ketimines 1-4.

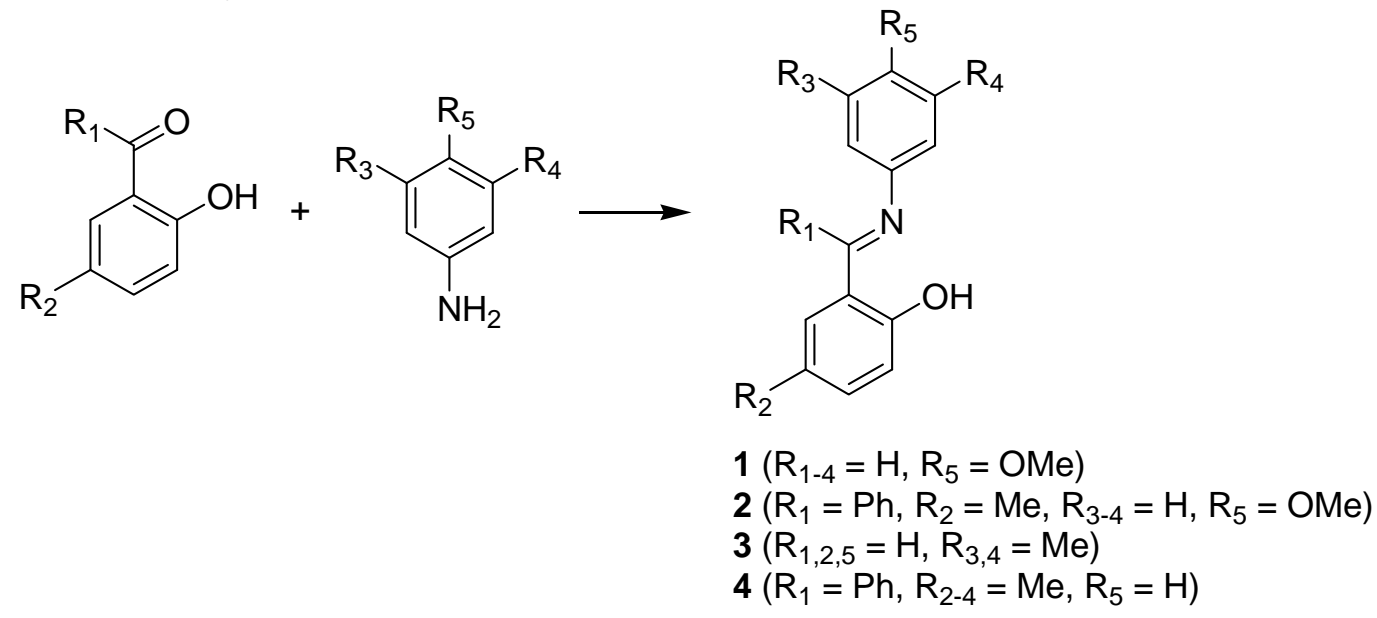

Scheme 2. Synthesis of 3,6-dibenzoylcatechol.<smiles>[R4]C(=O)C(O)C(=O)c1ccccc1</smiles>

Synthesis of $\boldsymbol{N}$-salicylidene-p-anisidine (1): $p$-Anisidine (1.0 g, $8.1 \mathrm{mmol})$ was added to salicylaldehyde $(850 \mu \mathrm{L}, 8.1 \mathrm{mmol})$ while stirring, resulting in immediate precipitation of a yellow solid. The product was filtered and recrystallized from $\mathrm{EtOH}$ yielding $1.2 \mathrm{~g}$ of $\mathbf{1}$ as light green/yellow flakes (5.3 mmol, 65\%).

Data for $\boldsymbol{N}$-salicylidene-p-anisidine (1): ${ }^{13} \mathrm{C} \mathrm{NMR}\left(100.6 \mathrm{MHz}, \mathrm{CDCl}_{3}\right) \delta 161.2,160.7$, 159.1, 141.6, 132.9, 132.2, 122.5, 119.6, 117.4, 114.8, 100.2, 55.8. ${ }^{1} \mathrm{H}$ NMR (400 MHz, $\left.\mathrm{CDCl}_{3}\right) \delta 13.42(\mathrm{bs}, 1 \mathrm{H}, \mathrm{OH}), 8.62(\mathrm{~s}, 1 \mathrm{H}$, imine), $7.38(\mathrm{~m}, 2 \mathrm{H}, \mathrm{Ar}), 7.29(\mathrm{~m}, 2 \mathrm{H}, \mathrm{Ar})$, $7.03\left(\mathrm{~d}, \mathrm{~J}_{\mathrm{HH}}=7.6 \mathrm{~Hz}, 1 \mathrm{H}, \mathrm{Ar}\right), 6.96(\mathrm{~m}, 3 \mathrm{H}, \mathrm{Ar}), 3.85\left(\mathrm{~s}, 3 \mathrm{H}, \mathrm{OCH}_{3}\right) . \mathrm{UV}$-vis $(\mathrm{MeOH})$

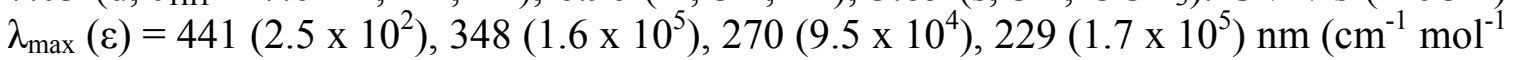
L). IR (neat) $v=1602,1569,1490,1456,1409,1279,1245,1186,1150,1028,836,738$ $\mathrm{cm}^{-1}$. ESI-MS $(\mathrm{MeOH}) m / z=228.2(\mathbf{1}+\mathrm{H})^{+}$. MP $=81{ }^{\circ} \mathrm{C}$. Anal. Calc'd for $\mathrm{C}_{14} \mathrm{H}_{13} \mathrm{NO}_{2}: \mathrm{C}$ $73.99 \%$, N 6.16\%, H 5.77\%; Found: C 73.92\%, N 6.12\%, H 5.86\%.

Synthesis of p-anisidine ketimine (2): A Schlenk tube charged with 2-benzoyl-4methylphenol $^{5}(100 \mathrm{mg}, 0.47 \mathrm{mmol})$ and $p$-anisidine $(116 \mathrm{mg}, 0.94 \mathrm{mmol})$ was heated to $110{ }^{\circ} \mathrm{C}$. After $1 \mathrm{~h}$, the dark brown solution was removed from heat and diluted with $\mathrm{MeOH}$. The solution was placed in the freezer for $12 \mathrm{~h}$ yielding $145 \mathrm{mg}$ of 2 as yellow crystals $(0.46 \mathrm{mmol}, 98 \%)$. 
Data for $\boldsymbol{p}$-anisidine ketimine (2): ${ }^{13} \mathrm{C} \mathrm{NMR}\left(100.6 \mathrm{MHz}, \mathrm{CDCl}_{3}\right) \delta 172.5,160.7,156.9$, $140.0,134.7,134.1,132.0,129.0,128.5,127.0,124.1,119.9,117.9,113.9,100.2,55.5$, 20.8. ${ }^{1} \mathrm{H}$ NMR $\left(400 \mathrm{MHz}, \mathrm{CDCl}_{3}\right) \delta 14.68(\mathrm{bs}, 1 \mathrm{H}, \mathrm{OH}), 7.37$ (m, 3H, Ar), 7.17 (m, 3H, Ar), $6.98\left(\mathrm{~d}, \mathrm{~J}_{\mathrm{HH}}=8.0 \mathrm{~Hz}, 1 \mathrm{H}, \operatorname{Ar}\right), 6.84(\mathrm{~s}, 1 \mathrm{H}, \operatorname{Ar}), 6.69(\mathrm{~m}, 4 \mathrm{H}, \operatorname{Ar}), 3.73(\mathrm{~s}, 3 \mathrm{H}$, $\left.\mathrm{OCH}_{3}\right), 2.16\left(\mathrm{~s}, 3 \mathrm{H}, \mathrm{CH}_{3}\right)$. UV-Vis $(\mathrm{MeOH}) \lambda_{\max }(\varepsilon)=349\left(7.2 \times 10^{4}\right), 231\left(1.9 \times 10^{5}\right) \mathrm{nm}$ $\left(\mathrm{cm}^{-1} \mathrm{~mol}^{-1} \mathrm{~L}\right)$. IR (neat) $v=2949,1740,1605,1567,1489,1458,1439,1234,1033,889$, $830,790,540 \mathrm{~cm}^{-1}$. ESI-MS $(\mathrm{MeOH}) \mathrm{m} / z=318.2(2+\mathrm{H})^{+} . \mathrm{MP}=162{ }^{\circ} \mathrm{C}$. Anal. Calc'd for $\mathrm{C}_{21} \mathrm{H}_{19} \mathrm{NO}_{2}$ : C 79.47\%, N 4.41\%, H 6.03\%; Found: C 79.40\%, N 4.45\%, H 5.98\%.

Synthesis of $N$-salicylidene-(3,5-dimethylaniline) (3): Salicylaldehyde (500 $\mu \mathrm{L}, 4.8$ mmol) and 3,5-dimethylaniline $(595 \mu \mathrm{L}, 4.8 \mathrm{mmol})$ were mixed in a test tube and heated to $150{ }^{\circ} \mathrm{C}$ for $1 \mathrm{~h}$. Upon cooling to room temperature, the crude mixture was washed through a small pad of silica gel (10:1, hexanes:ether). The solvent was removed under reduced pressure followed by heating under reduced pressure to remove excess salicylaldehyde. The remaining $535 \mathrm{mg}$ of yellow, viscous oil was compound 3 (2.4 mmol, 50\%).

Data for $\boldsymbol{N}$-salicylidene-(3,5-dimethylaniline) (3): $\left.{ }^{13} \mathrm{C} \mathrm{NMR} \mathrm{(100.6} \mathrm{MHz}, \mathrm{CDCl}_{3}\right) \delta$ $162.4,161.4,148.6,139.3,133.1,132.3,128.8,119.5,119.1,117.4,100.1,21.5 .{ }^{1} \mathrm{H}$ NMR (400 MHz, $\left.\mathrm{CDCl}_{3}\right) \delta 13.36(\mathrm{bs}, 1 \mathrm{H}, \mathrm{OH}), 8.63$ (s, 1H, imine), 7.39 (m, 2H, Ar), $7.04\left(\mathrm{~d}, \mathrm{~J}_{\mathrm{HH}} 8.0 \mathrm{~Hz}, 1 \mathrm{H}, \mathrm{Ar}\right), 6.95(\mathrm{~m}, 4 \mathrm{H}, \mathrm{Ar}), 2.38\left(\mathrm{~s}, 6 \mathrm{H}, \mathrm{CH}_{3}\right)$. UV-Vis $\left(\mathrm{CH}_{2} \mathrm{Cl}_{2}\right) \lambda_{\max }$ $(\varepsilon)=341\left(1.8 \times 10^{4}\right), 270\left(1.6 \times 10^{4}\right) \mathrm{nm}\left(\mathrm{cm}^{-1} \mathrm{~mol}^{-1} \mathrm{~L}\right)$. IR (neat) $v=3010,2981,2916$, 2863, 1619, 1605, 1590, 1572, 1495, 1458, 1276, 1206, 1147, 843, 751, 685, $583 \mathrm{~cm}^{-1}$. ESI-MS $(\mathrm{MeOH}) m / z=226.3(3+\mathrm{H})^{+}$. Anal. Calc'd for $\mathrm{C}_{15} \mathrm{H}_{15} \mathrm{NO}$ : C 79.97\%, N 6.22\%, H 6.71\%; Found: C 80.02\%, N 6.24\%, H 6.73\%.

Synthesis of 3,5-dimethylaniline ketimine (4): In a Schlenk tube, 2-benzoyl-4methylphenol (100 mg, $0.47 \mathrm{mmol})$ and 3,5-dimethylaniline $(88 \mu \mathrm{L}, 0.71 \mathrm{mmol})$ were mixed and heated to $140{ }^{\circ} \mathrm{C}$ under reduced pressure. After $30 \mathrm{~min}$, the temperature was reduced to $120{ }^{\circ} \mathrm{C}$ where it was kept for $21 \mathrm{~h}$. The crude yellow oil was purified by column chromatography in 10:1 hexanes:ether and then recrystallized from a chilled $\mathrm{MeOH}$ solution with a small amount of hexanes. Filtration of the yellow crystals gave 45 mg of compound 4 (0.14 mmol, 30\%).

Data for 3,5-dimethylaniline ketimine (4): ${ }^{13} \mathrm{C}$ NMR (100.6 $\left.\mathrm{MHz}, \mathrm{CDCl}_{3}\right) \delta 173.0$, $160.7,146.9,138.3,134.6,134.2,132.2,129.0,128.3,127.1,126.4,120.5,119.7,118.0$, 100.2, 21.4, 20.7. ${ }^{1} \mathrm{H}$ NMR (400 MHz, $\left.\mathrm{CDCl}_{3}\right) \delta 14.43(\mathrm{bs}, 1 \mathrm{H}, \mathrm{OH}), 7.35$ (m, 3H, Ar), $7.17(\mathrm{~m}, 3 \mathrm{H}, \mathrm{Ar}), 6.99$ (d, J $\left.\mathrm{HH}_{\mathrm{HH}} .0 \mathrm{~Hz}, 1 \mathrm{H}, \mathrm{Ar}\right) 6.85$ (s, 1H, Ar), 6.62 (s, 1H, Ar), 6.38 (s, $2 \mathrm{H}, \mathrm{Ar}), 2.17\left(\mathrm{~s}, 3 \mathrm{H}, \mathrm{CH}_{3}\right), 2.16\left(\mathrm{~s}, 6 \mathrm{H}, \mathrm{CH}_{3}\right)$. UV-Vis $\left(\mathrm{CH}_{2} \mathrm{Cl}_{2}\right) \lambda_{\max }(\varepsilon)=342\left(8.4 \times 10^{3}\right)$, $267\left(1.5 \times 10^{4}\right) \mathrm{nm}\left(\mathrm{cm}^{-1} \mathrm{~mol}^{-1} \mathrm{~L}\right)$. IR (neat) $v=3025,2917,1734,1604,1568,1488$, $1442,1332,1285,1234,1132,858,843,724,681 \mathrm{~cm}^{-1}$. ESI-MS $(\mathrm{MeOH}) \mathrm{m} / z=316.2$ $(4+\mathrm{H})^{+}$. $\mathrm{MP}=111{ }^{\circ} \mathrm{C}$. Anal. Calc'd for $\mathrm{C}_{22} \mathrm{H}_{21} \mathrm{NO}$ : C 83.78\%, N 4.44\%, H 6.71\%; Found: C 83.60\%, N 4.44\%, H 6.73\%. 
Data for tetraphenyl-o-benzodifuran (5): ${ }^{13} \mathrm{C}$ NMR $\left(100.6 \mathrm{MHz}, \mathrm{CDCl}_{3}\right) \delta 150.3$, $138.7,132.9,130.6,129.9,129.8,129.0,128.4,128.2,127.8,126.9,118.7,115.2 .{ }^{1} \mathrm{H}$ NMR (400 MHz, CDCl $) \delta 7.76\left(\mathrm{bd}, \mathrm{J}_{\mathrm{HH}} 6.9 \mathrm{~Hz}, 4 \mathrm{H}, \mathrm{Ar}\right), 7.56\left(\mathrm{bd}, \mathrm{J}_{\mathrm{HH}} 7.8 \mathrm{~Hz}, 4 \mathrm{H}, \mathrm{Ar}\right)$, 7.51 (bt, $\left.\mathrm{J}_{\mathrm{HH}} 7.3 \mathrm{~Hz}, 4 \mathrm{H}, \mathrm{Ar}\right), 7.45$ (m, 2H, Ar), 7.36 (m, 6H, Ar), 7.32 (s, 2H, Ar). UVVis $\left(\mathrm{CH}_{2} \mathrm{Cl}_{2}\right) \lambda_{\max }(\varepsilon)=340\left(3.8 \times 10^{4}\right), 263\left(3.8 \times 10^{4}\right) \mathrm{nm}\left(\mathrm{cm}^{-1} \mathrm{~mol}^{-1} \mathrm{~L}\right)$. IR (neat) $v=$ 1601, 1483, 1497, 1451, 1440, 1391, 1370, 1277, 1247, 1096, 1070, 944, 804, 767, 753, $694,681,646 \mathrm{~cm}^{-1}$. EI-MS $\mathrm{m} / z=462(5)^{+}$. MP $=240{ }^{\circ} \mathrm{C}$. Anal. Calc'd for $\mathrm{C}_{34} \mathrm{H}_{22} \mathrm{O}_{2}: \mathrm{C}$ $88.29 \%$, H 4.79\%; Found: C 88.04\%, H 4.82\%.

Data for 2,3-bis(benzoyloxy)-1,4-dibenzoylbenzene (5b): ${ }^{13} \mathrm{C}$ NMR $(100.6 \mathrm{MHz}$, $\left.\mathrm{CDCl}_{3}\right) \delta$ 193.1, 163.5, 141.8, 136.7, 135.9, 133.6, 133.4, 130.0, 128.5, 128.2, 127.9, 126.9. ${ }^{1} \mathrm{H}$ NMR $\left(300 \mathrm{MHz}, \mathrm{CDCl}_{3}\right) \delta 7.89\left(\mathrm{~d}, \mathrm{~J}_{\mathrm{HH}} 7.2 \mathrm{~Hz}, 4 \mathrm{H}, \mathrm{Ar}\right), 7.75\left(\mathrm{~d}, \mathrm{~J}_{\mathrm{HH}} 7.2 \mathrm{~Hz}\right.$, $4 \mathrm{H}, \mathrm{Ar}), 7.62(\mathrm{~s}, 2 \mathrm{H}, \mathrm{Ar}), 7.54\left(\mathrm{t}, \mathrm{J}_{\mathrm{HH}} 7.2 \mathrm{~Hz}, 2 \mathrm{H}, \mathrm{Ar}\right), 7.44(\mathrm{~m}, 6 \mathrm{H}, \mathrm{Ar}), 7.24\left(\mathrm{t}, \mathrm{J}_{\mathrm{HH}}=7.8\right.$ $\mathrm{Hz}, 4 \mathrm{H}, \mathrm{Ar})$. UV-Vis $\left(\mathrm{CH}_{2} \mathrm{Cl}_{2}\right) \lambda_{\max }(\varepsilon)=239\left(3.8 \times 10^{4}\right) \mathrm{nm}\left(\mathrm{cm}^{-1} \mathrm{~mol}^{-1} \mathrm{~L}\right)$. IR (neat) $v=$ $1748,1739,1660,1256,1225,1084,1051,708,699,690 \mathrm{~cm}^{-1}$. EI-MS $\mathrm{m} / z=526(5 \mathbf{b})^{+}$. $\mathrm{MP}=144{ }^{\circ} \mathrm{C}$. Anal. Calc'd for $\mathrm{C}_{34} \mathrm{H}_{22} \mathrm{O}_{6}$ : C $77.56 \%, \mathrm{H} 4.21 \%$; Found: $\mathrm{C} 77.56 \%, \mathrm{H}$ $4.27 \%$.

Data for 3,6-dibenzoylcatechol (6): ${ }^{13} \mathrm{C}$ NMR $\left(100.6 \mathrm{MHz}, \mathrm{CDCl}_{3}\right) \delta$ 201.0, 152.6, $137.4,132.8,129.5,128.6,122.3,121.3 .{ }^{1} \mathrm{H}$ NMR $\left(400 \mathrm{MHz}, \mathrm{CDCl}_{3}\right) \delta 11.68(\mathrm{~s}, 2 \mathrm{H}$, $\mathrm{OH}), 7.75\left(\mathrm{~d}, \mathrm{~J}_{\mathrm{HH}} 6.8 \mathrm{~Hz}, 4 \mathrm{H}, \mathrm{Ar}\right), 7.63\left(\mathrm{t}, \mathrm{J}_{\mathrm{HH}} 7.6 \mathrm{~Hz}, 2 \mathrm{H}, \mathrm{Ar}\right), 7.53$ (t, $\mathrm{J}_{\mathrm{HH}} 7.6 \mathrm{~Hz}, 4 \mathrm{H}$, Ar), $7.12(\mathrm{~s}, 2 \mathrm{H}, \mathrm{Ar})$. UV-Vis $\left(\mathrm{CH}_{2} \mathrm{Cl}_{2}\right) \lambda_{\max }(\varepsilon)=405\left(2.5 \times 10^{3}\right), 301\left(2.1 \times 10^{4}\right) \mathrm{nm}$ $\left(\mathrm{cm}^{-1} \mathrm{~mol}^{-1} \mathrm{~L}\right)$. IR (neat) $v=1611,1599,1417,1331,1317,1299,1233,1184,988,939$, $861,839,793,766,625,690 \mathrm{~cm}^{-1}$. EI-MS $m / z=318(6)^{+}, 241(6-\mathrm{Ph})^{+}, 212(6-\mathrm{COPh})^{+}$. $\mathrm{MP}=160{ }^{\circ} \mathrm{C}$. Anal. Calc'd for $\mathrm{C}_{20} \mathrm{H}_{14} \mathrm{O}_{4}$ : C 75.46\%, H 4.43\%; Found: C $75.05 \%, \mathrm{H}$ $4.48 \%$.

Synthesis of model diketimine (8): $p$-Anisidine $(40 \mathrm{mg}, 0.32 \mathrm{mmol})$ was added to a suspension of 3,6-dibenzoylcatechol, 6, (50 mg, $0.15 \mathrm{mmol})$ in $15 \mathrm{~mL}$ of $\mathrm{MeOH}$ and the reaction was heated to $70{ }^{\circ} \mathrm{C}$. After $12 \mathrm{~h}$, the reaction was cooled to RT. Upon standing, yellow crystals formed on the walls. The crystals were isolated by filtration giving $65 \mathrm{mg}$ of compound $8(0.12 \mathrm{mmol}, 82 \%)$. The crystals obtained by this method were subjected to a single-crystal X-ray diffraction study.

Data for model diketimine (8): ${ }^{13} \mathrm{C}$ NMR $\left(100.6 \mathrm{MHz}, \mathrm{CDCl}_{3}\right) \delta 171.9,157.3,153.3$, 139.5, 134.7, 129.1, 128.9, 128.6, 124.5, 121.1, 119.4, 114.0, 55.5. ${ }^{1} \mathrm{H}$ NMR (400 MHz, $\left.\mathrm{CDCl}_{3}\right) \delta 15.30(\mathrm{~s}, 2 \mathrm{H}, \mathrm{OH}), 7.34(\mathrm{~m}, 6 \mathrm{H}, \mathrm{Ar}) 7.16(\mathrm{~m}, 4 \mathrm{H}, \mathrm{Ar}), 6.74\left(\mathrm{t}, \mathrm{J}_{\mathrm{HH}} 9.5 \mathrm{~Hz}, 4 \mathrm{H}\right.$, Ar) $6.71\left(\mathrm{t}, \mathrm{J}_{\mathrm{HH}} 9.5 \mathrm{~Hz}, 4 \mathrm{H}, \mathrm{Ar}\right), 6.39(\mathrm{~s}, 2 \mathrm{H}, \mathrm{Ar}), 3.74\left(\mathrm{~s}, 6 \mathrm{H}, \mathrm{OCH}_{3}\right)$. UV-Vis $\left(\mathrm{CH}_{2} \mathrm{Cl}_{2}\right)$ $\lambda_{\max }(\varepsilon)=374\left(1.6 \times 10^{4}\right), 300\left(1.8 \times 10^{4}\right), 240\left(2.2 \times 10^{4}\right) \mathrm{nm}^{-1}\left(\mathrm{~cm}^{-1} \mathrm{~mol}^{-1} \mathrm{~L}\right)$. IR (neat) $v$ $=2837,1604,1491,1429,1328,1240,1175,1028,887,832,699,565 \mathrm{~cm}^{-1}$. ESI-MS $(\mathrm{MeOH}) \mathrm{m} / z=529.3(\mathbf{8}+\mathrm{H})^{+}$. MP $=233{ }^{\circ} \mathrm{C}$. Anal. Calc'd for $\mathrm{C}_{34} \mathrm{H}_{28} \mathrm{~N}_{2} \mathrm{O}_{4}: \mathrm{C} 77.25 \%$, N $5.30 \%$, H 5.34\%; Found: C 76.99\%, N 5.30\%, H 5.39\%.

Synthesis of 2:1 dipentyloxydiamine:3,6-dibenzoylcatechol (10): In a Schlenk flask, 4,5-diamino-1,2-dipentyloxybenzene, $\mathbf{9 a},(500 \mathrm{mg}, 1.8 \mathrm{mmol})$ was dissolved in a mixture of degassed toluene $(20 \mathrm{~mL})$ and piperidine $(200 \mu \mathrm{L}, 2.0 \mathrm{mmol})$. Addition of 3,6- 
dibenzoylcatechol, 6, $(282 \mathrm{mg}, 0.89 \mathrm{mmol})$ to the Schlenk flask resulted in the immediate evolution of a deep red color. The solution was heated to reflux for $12 \mathrm{~h}$ under $\mathrm{N}_{2}$. Upon cooling to RT, hexanes $(80 \mathrm{~mL})$ was added to the reaction flask resulting in the formation of a red precipitate that was isolated by filtration to give $250 \mathrm{mg}$ of $\mathbf{1 0}(0.30 \mathrm{mmol}, 33 \%)$.

Data for 2:1 dipentyloxydiamine:3,6-dibenzoylcatechol (10): ${ }^{13} \mathrm{C}$ NMR $(100.6 \mathrm{MHz}$, $\left.\mathrm{CDCl}_{3}\right) \delta 171.3,152.5,148.9,141.1,135.6,135.0,129.2,128.8,124.8,121.6,119.6$, $110.8,102.0,70.3,69.3,29.1,29.0,28.4,28.3,22.7,22.6,14.3,14.2 .{ }^{1} \mathrm{H}$ NMR $(400 \mathrm{MHz}$, $\left.\mathrm{CDCl}_{3}\right) \delta 15.20(\mathrm{~s}, 2 \mathrm{H}, \mathrm{OH}), 7.35(\mathrm{~m}, 6 \mathrm{H}, \mathrm{Ar}), 7.21(\mathrm{~m}, 4 \mathrm{H}, \mathrm{Ar}), 6.44(\mathrm{~s}, 2 \mathrm{H}, \mathrm{Ar}), 6.30(\mathrm{~s}$, $2 \mathrm{H}, \mathrm{Ar}), 5.88(\mathrm{~s}, 2 \mathrm{H}, \mathrm{Ar}), 3.89\left(\mathrm{t}, \mathrm{J}_{\mathrm{HH}} 6.9 \mathrm{~Hz}, 4 \mathrm{H}, \mathrm{OCH}_{2}\right), 3.83\left(\mathrm{bs}, 4 \mathrm{H}, \mathrm{NH}_{2}\right), 3.37\left(\mathrm{t}, \mathrm{J}_{\mathrm{HH}}\right.$ $\left.6.9 \mathrm{~Hz}, 4 \mathrm{H}, \mathrm{OCH}_{2}\right), 1.77\left(\mathrm{~m}, 4 \mathrm{H}, \mathrm{OCH}_{2} \mathrm{CH}_{2}\right) 1.51\left(\mathrm{~m}, 4 \mathrm{H}, \mathrm{OCH}_{2} \mathrm{CH}_{2}\right), 1.39(\mathrm{~m}, 8 \mathrm{H}$, alkyl), $1.28\left(\mathrm{~m}, 8 \mathrm{H}\right.$, alkyl), $0.91\left(\mathrm{~m}, 12 \mathrm{H}, \mathrm{CH}_{3}\right)$. UV-Vis $\left(\mathrm{CH}_{2} \mathrm{Cl}_{2}\right) \lambda_{\max }(\varepsilon)=461(1.7 \mathrm{x}$ $\left.10^{4}\right), 309\left(4.0 \times 10^{4}\right) \mathrm{nm}\left(\mathrm{cm}^{-1} \mathrm{~mol}^{-1} \mathrm{~L}\right)$. IR (neat) $v=3319,2954,2930,2858,1608,1584$, $1506,1465,1422,1328,1261,1213,1124,735,697 \mathrm{~cm}^{-1}$. EI-MS $\mathrm{m} / z=842(\mathbf{1 0})^{+}, 824$ $\left(\mathbf{1 0}-\mathrm{H}_{2} \mathrm{O}\right)^{+}, 766(\mathbf{1 0}-\mathrm{Ph})^{+}$. MP $=173{ }^{\circ} \mathrm{C}$. Anal. Calc'd for $\mathrm{C}_{52} \mathrm{H}_{66} \mathrm{~N}_{4} \mathrm{O}_{6}: \mathrm{C} \quad 74.08 \%, \mathrm{~N}$ $6.65 \%$, H 7.89\%; Found: C 74.41\%, N 6.76\%, H 7.82\%.

Synthesis of 2:1 dipentyloxydiamine:4,6-dibenzoylresorcinol (11a): In a Schlenk tube, under nitrogen, 4,6-dibenzoylresorcinol, 7 (200 $\mathrm{mg}, 0.63 \mathrm{mmol}$ ), and 4,5-diamino-1,2dipentyloxybenzene, 9a, $(380 \mathrm{mg}, 1.4 \mathrm{mmol})$ were mixed. With a heat gun, the mixture was heated to approximately $210{ }^{\circ} \mathrm{C}$ for 15 min until gas evolution was no longer observed from the dark red liquid. After cooling to room temperature, the dark red solid was dissolved in $\mathrm{CH}_{2} \mathrm{Cl}_{2}$ and concentrated under vacuum. A yellow precipitate formed upon addition of $\mathrm{MeOH}$. The precipitate was isolated by filtration yielding $117 \mathrm{mg}$ of 11a (0.14 mmol, 22\%).

Data for 2:1 dipentyloxydiamine:4,6-dibenzoylresorcinol (11a): ${ }^{13} \mathrm{C}$ NMR (100.6 $\left.\mathrm{MHz}, \mathrm{CDCl}_{3}\right) \delta 172.9,169.0,149.2,142.2,139.3,135.7,135.3,129.8,129.2,129.1$, $125.7,114.3,112.3,106.0,103.1,71.1,70.1,29.9,29.7,29.1,29.0,23.4,23.3,15.0 .{ }^{1} \mathrm{H}$ NMR (400 MHz, CDCl $): \delta 15.74(\mathrm{~s}, 2 \mathrm{H}, \mathrm{OH}), 7.04(\mathrm{~m}, 10 \mathrm{H}, \mathrm{Ar}), 6.80(\mathrm{~s}, 1 \mathrm{H}, \mathrm{Ar}), 6.57$ (s, $1 \mathrm{H}, \mathrm{Ar}), 6.24$ (s, 2H, Ar), 5.81 (s, $2 \mathrm{H}, \mathrm{Ar}), 3.85\left(\mathrm{t}, 4 \mathrm{H}, \mathrm{OCH}_{2}\right), 3.66\left(\mathrm{~s}, 4 \mathrm{H}, \mathrm{NH}_{2}\right), 3.34$ (t, $\left.4 \mathrm{H}, \mathrm{OCH}_{2}\right), 1.80-0.80\left(\mathrm{~m}, 36 \mathrm{H}\right.$, alkyl). UV-Vis $\left(\mathrm{CH}_{2} \mathrm{Cl}_{2}\right) \lambda_{\max }(\varepsilon)=394\left(8.9 \times 10^{4}\right)$, $306\left(1.1 \times 10^{5}\right), 243\left(8.5 \times 10^{4}\right) \mathrm{nm}\left(\mathrm{cm}^{-1} \mathrm{~mol}^{-1} \mathrm{~L}\right)$. IR (neat) $v=3465,3373,2954,2930$, 2869, 1619, 1568, 1507, 1469, 1331, 1250, 1198, 1113, 988, 918, 846, $698 \mathrm{~cm}^{-1}$. MALDI-TOF MS (dithranol matrix) $\mathrm{m} / z=842.2(\mathbf{1 1 a})^{+}$. MP $=208-212{ }^{\circ} \mathrm{C}$. Anal. Calc'd for $\mathrm{C}_{52} \mathrm{H}_{66} \mathrm{~N}_{4} \mathrm{O}_{6}$ : C 74.08\%, N 6.65\%, H 7.89\%; Found: C 74.06\%, N 6.58\%, H 7.78\%.

Synthesis of 2:1 dihexyloxydiamine:4,6-dibenzoylresorcinol (11b): Under $\mathrm{N}_{2}$, a Schlenk tube was charged with 4,6-dibenzoylresorcinol, 7, (100 $\mathrm{mg}, 0.32 \mathrm{mmol})$ and 4,5diamino-1,2-dihexyloxybenzene, $9 \mathbf{b},(210 \mathrm{mg}, 0.68 \mathrm{mmol})$ and heated with a heat gun to approximately $210^{\circ} \mathrm{C}$ for 15 mins until gas evolution was no longer observed. After cooling to room temperature, the dark red solid was transferred with $\mathrm{CH}_{2} \mathrm{Cl}_{2}$ and concentrated under vacuum. Suspension of the remaining solid in $\mathrm{MeOH}$ followed by filtration gave $92 \mathrm{mg}$ of $\mathbf{1 1 b}$ as a yellow powder $(0.10 \mathrm{mmol}, 32 \%)$. 
Data for 2:1 dihexyloxydiamine:4,6-dibenzoylresorcinol (11b): ${ }^{13} \mathrm{C}$ NMR $(100.6 \mathrm{MHz}$, $\left.\mathrm{CDCl}_{3}\right) \delta 172.7,168.9,149.1,142.9,139.0,135.5,135.1,129.7,129.2,129.1,125.7$, 114.3, 114.1, 112.1, 105.0, 103.1, 78.2, 71.1, 70.1, 32.6, 32.5, 30.2, 30.0, 26.6, 26.5, 23.6, 15.0. ${ }^{1} \mathrm{H}$ NMR $\left(400 \mathrm{MHz}, \mathrm{CDCl}_{3}\right) \delta 15.76(\mathrm{~s}, 2 \mathrm{H}, \mathrm{OH}), 7.06(\mathrm{~m}, 10 \mathrm{H}, \mathrm{Ar}), 6.83(\mathrm{~s}, 1 \mathrm{H}$, Ar), 6.59 (s, 1H, Ar), $6.26(\mathrm{~s}, 2 \mathrm{H}, \mathrm{Ar}), 5.84(\mathrm{~s}, 2 \mathrm{H}, \mathrm{Ar}), 3.87\left(\mathrm{t}, 4 \mathrm{H}, \mathrm{OCH}_{2}\right), 3.68(\mathrm{~s}, 4 \mathrm{H}$, $\left.\mathrm{NH}_{2}\right), 3.37$ (t, 4H, OCH$)_{2}, 1.80-0.80\left(\mathrm{~m}, 44 \mathrm{H}\right.$, alkyl). UV-Vis $\left(\mathrm{CH}_{2} \mathrm{Cl}_{2}\right) \lambda_{\max }(\varepsilon)=422$ $\left(2.0 \times 10^{4}\right), 283\left(4.1 \times 10^{4}\right) \mathrm{nm}\left(\mathrm{cm}^{-1} \mathrm{~mol}^{-1} \mathrm{~L}\right)$. IR (neat) $v=3466,3374,2954,2927$, $2858,1618,1569,1506,1469,1331,1249,1198,916,847,698 \mathrm{~cm}^{-1}$. MALDI-TOF MS (dithranol matrix) $\mathrm{m} / \mathrm{z}=900.1\left(\mathbf{1 1 b}+\mathrm{H}^{+}\right) . \mathrm{MP}=182-184{ }^{\circ} \mathrm{C}$. Anal. Calc'd for $\mathrm{C}_{56} \mathrm{H}_{74} \mathrm{~N}_{4} \mathrm{O}_{6}$ : N 6.23\%, C 74.80\%, H 8.29\%; Found: N 6.06\%, C 73.98\%, H 8.15\%.

Synthesis of [2+2] macrocycle (12): In a Schlenk flask, compound 10 (56.4 mg, 0.07 $\mathrm{mmol})$, 4,6-diformylresorcinol $(11.1 \mathrm{mg}, 0.07 \mathrm{mmol})$, and piperidine $(40 \mu \mathrm{L})$, were dissolved in $60 \mathrm{~mL}$ of dry $\mathrm{CH}_{3} \mathrm{CN}: \mathrm{CHCl}_{3}(1: 2)$. The flask was fit with a condenser and the reaction mixture was refluxed for $16 \mathrm{~h}$ under $\mathrm{N}_{2}$. After cooling to room temperature, the solvent was removed under reduced pressure. The remaining solids were suspended in $\mathrm{MeOH}$ and filtered yielding a red powder. The crude product was recrystallized from $\mathrm{CH}_{2} \mathrm{Cl}_{2} / \mathrm{MeOH}$ giving $56 \mathrm{mg}$ of macrocycle $12(0.03 \mathrm{mmol} 41 \%)$.

Data for [2+2] macrocycle (12): ${ }^{13} \mathrm{C}$ NMR $\left(100.6 \mathrm{MHz}, \mathrm{CDCl}_{3}\right) \delta 175.8,167.1,158.4$, 153.9, 149.4, 147.8, 137.5, 137.2, 135.9, 132.4, 129.7, 128.7, 121.7, 120.8, 114.4, 109.7, 106.1, 104.1, 78.2, 71.2, 70.8, 30.0, 29.8, 29.2, 29.1, 23.5, 23.4, 15.0. ${ }^{1} \mathrm{H}$ NMR (400 MHz, $\left.\mathrm{CD}_{2} \mathrm{Cl}_{2}\right) \delta 14.53(\mathrm{~s}, 4 \mathrm{H}, \mathrm{OH}), 14.31(\mathrm{~s}, 4 \mathrm{H}, \mathrm{OH}), 8.57$ (s, 4H, imine), 7.49 (s, $\left.2 \mathrm{H}, \mathrm{Ar}\right)$, 7.20 (m, 20H, Ar), 6.80 (s, 4H, Ar), 6.54 (s, 2H, Ar), 6.45 (s, 4H, Ar), 6.19 (s, 4H, Ar), $3.95\left(\mathrm{t}, 8 \mathrm{H}, \mathrm{OCH}_{2}\right), 3.68\left(\mathrm{t}, 8 \mathrm{H}, \mathrm{OCH}_{2}\right), 1.90-0.90\left(\mathrm{~m}, 72 \mathrm{H}\right.$, alkyl). UV-Vis $\left(\mathrm{CH}_{2} \mathrm{Cl}_{2}\right) \lambda_{\max }$ $(\varepsilon)=420\left(1.68 \times 10^{5}\right), 284\left(2.8 \times 10^{5}\right) \mathrm{nm}\left(\mathrm{cm}^{-1} \mathrm{~mol}^{-1} \mathrm{~L}\right)$. IR (neat) $v=2952,2928,2859$, $1624,1604,1588,1497,1330,1257,1176,1155,849,700 \mathrm{~cm}^{-1}$. MALDI-TOF MS (dithranol matrix) $\mathrm{m} / z=1946.4(\mathbf{1 2})^{+}, 1968.5(\mathbf{1 2}+\mathrm{Na})^{+}$. HRMS (ESI) calc'd for ${ }^{12} \mathrm{C}_{119}{ }^{13} \mathrm{CH}_{136} \mathrm{~N}_{8} \mathrm{O}_{16}: 1946.0108$; found: $m / z=1946.0184\left[\mathrm{M}^{+}\right]$. MP $=$dec. at $270{ }^{\circ} \mathrm{C}$.

Synthesis of [2+2] macrocycle (13): Prepared by the same method as macrocycle 12 using 11a (24 mg, $0.03 \mathrm{mmol}), 3,6$-diformylcatechol ( $4.7 \mathrm{mg}, 0.03 \mathrm{mmol})$, and piperidine $(40 \mu \mathrm{L})$. The crude product was recrystallized from $\mathrm{CH}_{2} \mathrm{Cl}_{2} / \mathrm{MeOH}$ giving $20 \mathrm{mg}$ of macrocycle $13(0.01 \mathrm{mmol}, 35 \%)$.

Data for [2+2] macrocycle (13): ${ }^{13} \mathrm{C}$ NMR $\left(100.6 \mathrm{MHz}, \mathrm{CDCl}_{3}\right) \delta 173.5,169.0,161.5$, $150.9,149.2,147.8,139.9,135.3,134.3,129.3,129.2,128.8,122.2,121.8,113.7,110.2$, 106.4, 106.3, 77.7, 70.6, 70.0, 29.9, 29.6, 29.1, 29.0, 23.5, 23.4, 15.0. ${ }^{1} \mathrm{H}$ NMR (400 MHz, $\left.\mathrm{CDCl}_{3}\right) \delta 15.20(\mathrm{~s}, 4 \mathrm{H}, \mathrm{OH}), 12.89(\mathrm{~s}, 4 \mathrm{H}, \mathrm{OH}), 8.35$ (s, 4H, imine), 6.95 (m, 26H, Ar), $6.61(\mathrm{~s}, 4 \mathrm{H}, \mathrm{Ar}), 6.52(\mathrm{~s}, 2 \mathrm{H}, \mathrm{Ar}), 6.14(\mathrm{~s}, 4 \mathrm{H}, \mathrm{Ar}), 3.91\left(\mathrm{t}, 8 \mathrm{H}, \mathrm{OCH}_{2}\right), 3.57(\mathrm{t}, 8 \mathrm{H}$, $\left.\mathrm{OCH}_{2}\right), 1.80-0.80\left(\mathrm{~m}, 72 \mathrm{H}\right.$, alkyl). UV-Vis $\left(\mathrm{CH}_{2} \mathrm{Cl}_{2}\right) \lambda_{\max }(\varepsilon)=439\left(1.54 \times 10^{5}\right), 388$

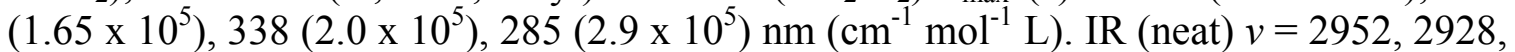
$2858,1615,1558,1305,1259,1183,992,849,740,698 \mathrm{~cm}^{-1}$. MALDI-TOF MS (dithranol matrix) $\mathrm{m} / \mathrm{z}=1946.4(\mathbf{1 3})^{+} . \mathrm{MP}=245-250{ }^{\circ} \mathrm{C}$. Anal. Calc'd for $\mathrm{C}_{120} \mathrm{H}_{136} \mathrm{~N}_{8} \mathrm{O}_{16} \cdot 2 \mathrm{MeOH}$ : N 5.57\%, C 72.88\%, H 7.22\%; Found: N 5.63\%, C 72.32\%, H $6.88 \%$. 
Synthesis of [2+2] macrocycle (14): Compound 11b (40 mg, $0.045 \mathrm{mmol})$ and 4,6diformylresorcinol $(7.4 \mathrm{mg}, 0.045 \mathrm{mmol})$ were dissolved in a mixture of chloroform (7 $\mathrm{mL})$ and acetonitrile $(9 \mathrm{~mL})$ under $\mathrm{N}_{2}$. The orange solution was stirred at reflux $\left(85^{\circ} \mathrm{C}\right)$ for $24 \mathrm{~h}$. Orange precipitate was observed after stirring overnight. After cooling to room temperature, the precipitate was isolated by filtration yielding $12 \mathrm{mg}$ of macrocycle 14 (0.012 mmol, 26\%).

Data for macrocycle (14): ${ }^{13} \mathrm{C}$ NMR $\left(100.6 \mathrm{MHz}, \mathrm{CD}_{2} \mathrm{Cl}_{2}\right) \delta 174.4,168.2,166.7,157.8$, $148.9,147.5,139.5,137.1,136.2,134.6,132.5,129.0,128.6,128.2,113.9,113.4,109.2$, 105.3, 105.1, 103.0, 70.2, 69.8, 32.2, 32.1, 29.9, 29.5, 26.3, 26.1, 23.2, 14.4. ${ }^{1} \mathrm{H}$ NMR $\left(300 \mathrm{MHz}, \mathrm{CD}_{2} \mathrm{Cl}_{2}\right) \delta 14.97$ (s, $\left.4 \mathrm{H}, \mathrm{OH}\right), 14.27(\mathrm{~s}, 4 \mathrm{H}, \mathrm{OH}), 8.43$ (s, 4H, imine), 7.36 (s, $2 \mathrm{H}, \mathrm{Ar}), 6.99(\mathrm{~m}, 12 \mathrm{H}, \mathrm{Ar}), 6.86(\mathrm{~s}, 4 \mathrm{H}, \mathrm{Ar}), 6.83$ (s, 4H, Ar), 6.73 (s, 4H, Ar), 6.68 (d, $4 \mathrm{H}, \mathrm{Ar}), 6.48(\mathrm{~s}, 2 \mathrm{H}, \mathrm{Ar}), 6.14(\mathrm{~s}, 4 \mathrm{H}, \mathrm{Ar}), 3.92\left(\mathrm{t}, 8 \mathrm{H}, \mathrm{OCH}_{2}\right), 3.67\left(\mathrm{t}, 8 \mathrm{H}, \mathrm{OCH}_{2}\right), 1.75$ (m, 8H, $\left.\mathrm{CH}_{2}\right), 1.60\left(\mathrm{~m}, 8 \mathrm{H}, \mathrm{CH}_{2}\right), 1.42\left(\mathrm{~m}, 8 \mathrm{H}, \mathrm{CH}_{2}\right), 1.31\left(\mathrm{~m}, 40 \mathrm{H}, \mathrm{CH}_{2}\right), 0.90(\mathrm{~m}, 24 \mathrm{H}$, $\left.\mathrm{CH}_{3}\right)$. UV-Vis $\left(\mathrm{CH}_{2} \mathrm{Cl}_{2}\right) \lambda_{\max }(\varepsilon)=283\left(1.19 \times 10^{5}\right), 388\left(8.13 \times 10^{4}\right) \mathrm{nm}\left(\mathrm{cm}^{-1} \mathrm{~mol}^{-1} \mathrm{~L}\right)$. IR (KBr) $v=3459,3060,2954,2930,2869,2859,1721,1625,1604,1585,1504,1469$, 1444, 1388, 1378, 1365, 1328, 1260, 1210, 1179, 1151, 1135, 1104, 1074, 1044, 1013, $962,916,890,850,774,741,725,699,647,610,543 \mathrm{~cm}^{-1}$. MALDI-TOF MS (dithranol matrix) $m / z=2059.6(\mathbf{1 4}+\mathrm{H})^{+}$. MP $>270{ }^{\circ} \mathrm{C}$. Anal. Calc'd for $\mathrm{C}_{128} \mathrm{H}_{152} \mathrm{~N}_{8} \mathrm{O}_{16}:$ C $74.68 \%$, H 7.44\%, N 5.44\%; Found: C 74.44\%, H 7.39\%, N 5.32\%.

X-ray Crystallography: Crystals of model diketimine 8 suitable for X-ray diffraction were obtained from hot $\mathrm{MeOH}$. X-ray crystal data for 8: $\mathrm{C}_{34} \mathrm{H}_{28} \mathrm{~N}_{2} \mathrm{O}_{4}, \mathrm{M}_{\mathrm{w}}=528.58 \mathrm{~g}$ $\mathrm{mol}^{-1}$, orange prism $\left(0.30 \times 0.30 \times 0.20 \mathrm{~mm}^{3}\right)$, orthorhombic, space group Pbca (\#61), a $=16.1351(9), \mathrm{b}=15.7795(12), \mathrm{c}=21.2316(15) \AA, \alpha=\beta=\gamma=90^{\circ}, V=5405.6(6) \AA^{3}, Z$ $=8, \rho_{\text {calcd }}=1.299 \mathrm{~g} \mathrm{~cm}^{-3}, \mathrm{~F}_{000}=2224, \mathrm{Mo}_{\mathrm{K} \alpha}$ radiation, $\lambda=0.71073 \AA, \mathrm{T}=173(2) \mathrm{K}$, $2 \theta_{\max }=44.94^{\circ}, 29621$ reflections collected, 5536 were unique $\left(\mathrm{R}_{\text {int }}=0.0609\right)$. Final $\mathrm{GoF}$ $=0.931, \mathrm{R}_{1}=0.0437, \mathrm{wR}_{2}=0.1013, \mathrm{R}$ indices based on 3287 reflections with $I>2 \sigma(I)$. The structure was solved by direct methods ${ }^{8}$ and the refinement was performed using SHELXL- $97^{9}$.All non-hydrogen atoms were refined anisotropically. All hydrogen atoms were located through difference mapping and refined isotropically.

Kinetics: An NMR tube was charged with $500 \mu \mathrm{L}$ of a $10 \mathrm{mmol} \mathrm{L}^{-1}$ solution of $\mathrm{N}$ salicylidene-p-anisidine, $\mathbf{1}$, in $\mathrm{CD}_{3} \mathrm{CN}\left(2.3 \mathrm{mg}\right.$ in $1.0 \mathrm{~mL}$ of $\left.\mathrm{CD}_{3} \mathrm{CN}\right)$. Two molar equiv of 3,5-dimethylaniline was introduced to the NMR tube via the addition of $60 \mu \mathrm{L}$ of a 160 mmol L-1 solution $\left(20 \mu \mathrm{L}\right.$ of 3,5-dimethylaniline in $1 \mathrm{~mL}$ of $\left.\mathrm{CD}_{3} \mathrm{CN}\right)$. No change was observed at $20^{\circ} \mathrm{C}$ over $20 \mathrm{~min}$.

The NMR spectrometer was preheated to $57{ }^{\circ} \mathrm{C}$ followed by introduction of the same sample. Resonances assigned to free $p$-anisidine were observed after $3 \mathrm{~min}$ indicating imine exchange was occurring. The initial $172 \mathrm{~s}$ allowed the sample to reach the desired temperature and was subtracted from the total time. Ten spectra were collected over the course of $1.3 \mathrm{~h}$. The concentration of $p$-anisidine was determined by integration of the ${ }^{1} \mathrm{H}$ NMR resonances and the experimental rate of exchange was found to be $0^{\text {th }}$ order with respect to $p$-anisidine until 20 mins had elapsed (Figure S1). After 20 mins the reverse reaction began competing, leading to a deviation from the initially observed $0^{\text {th }}$ order rate. 
At $57{ }^{\circ} \mathrm{C}$ the same experiment was attempted on $p$-anisidine ketimine 2. No exchange was observed over $36 \mathrm{~h}$. To ensure this was a kinetic effect and not a thermodynamic one, 3,5-dimethylaniline ketimine 4 was exposed to 2 equiv of $p$ anisidine in $\mathrm{CD}_{3} \mathrm{CN}$ at $57{ }^{\circ} \mathrm{C}$. No exchange was observed over $1.5 \mathrm{~h}$ for this reaction either.

${ }^{1}$ Ibrahim, M. N.; Hamad, K. J.; Al-Joroshi, S. H. Asian J. Chem. 2006, 18, 2404-2406.

2 (a) Dischendorfer, O.; Limontschew, W. Monat. fuer Chem. 1949, 80, 741-748. (b)

Limontschew, W.; Wiesenberger, E. Monat. fuer Chem. 1952, 83, 137-143.

${ }^{3}$ Abdul-Aziz, M.; Auping, J. V.; Meador, M. A. J. Org. Chem. 1995, 60, 1303-1308.

${ }^{4}$ Gallant, A. J.; Hui, J. K.-H.; Zahariev, F.; Wang, Y. A.; MacLachlan, M. J. J. Org. Chem. 2005, 70, 7936.

${ }^{5}$ Wei, J.-F.; Chen, G.; Shi, X.-Y.; Lei, N. Shenyang Huagong Xueyuan Xuebao 2004, 18, 259-262.

${ }^{6}$ Akine, S.; Taniguchi, T.; Nabeshima, T. Tetrahedron Lett. 2001, 42, 8861-8864.

${ }^{7}$ Worden, L. R.; Kaufman, K. D.; Smith, P. J.; Widiger, G. N. J. Chem. Soc., Chem. Commun. 1970, 227-230.

${ }^{8}$ SIR2002: Burla, M. C., Camalli, M., Carrozzini, B., Cascarano, G. L., Giacovazzo, C., Polidori, G., Spagna, R. (2003). J. Appl. Cryst. 2003, 36, 1103.

${ }^{9}$ Sheldrick, G. M. SHELXL-97. Programs for Crystal Structure Analysis (Release 97-2). University of Göttingen, Germany (1997). 


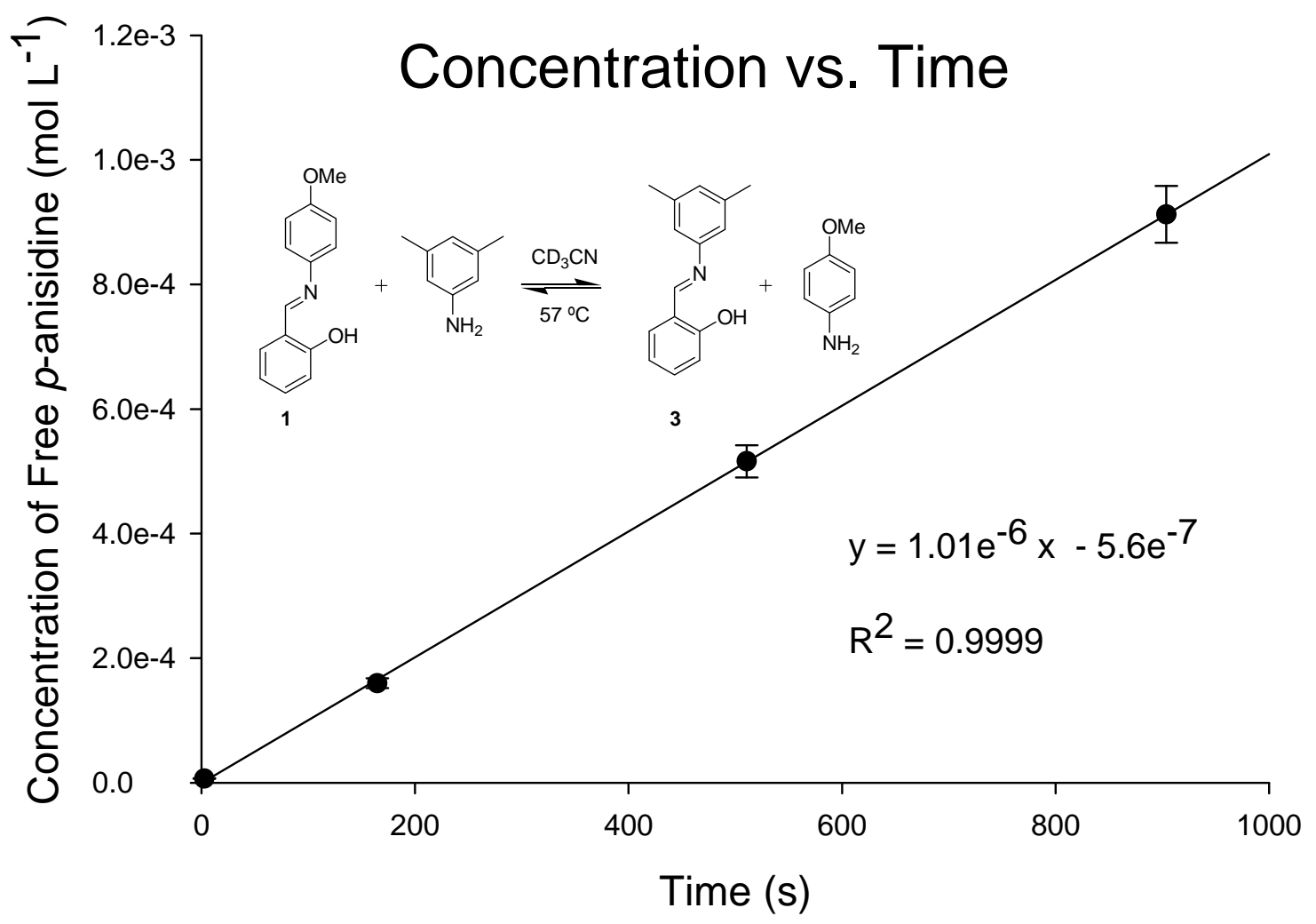

Figure S1. Concentration of free $p$-anisidine vs. time upon introduction of 2 equiv of 3,5dimethylaniline to $N$-salicylidene- $p$-anisidine, $\mathbf{1}$, at $57^{\circ} \mathrm{C}$.

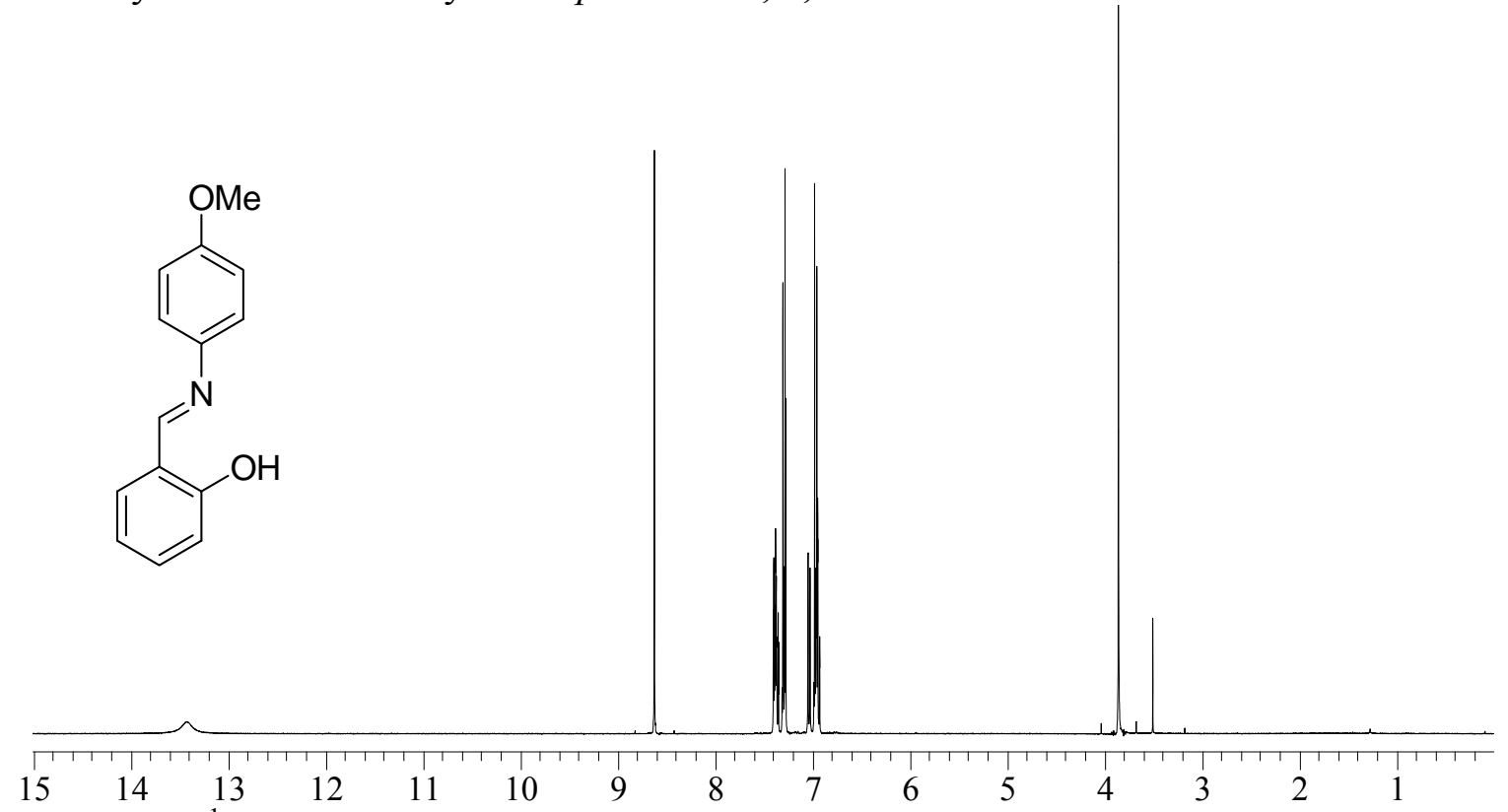

Figure S2. ${ }^{1} \mathrm{H}$ NMR spectrum of $N$-salicylidene- $p$-anisidine, 1 , in $\mathrm{CDCl}_{3}(400 \mathrm{MHz})$. 


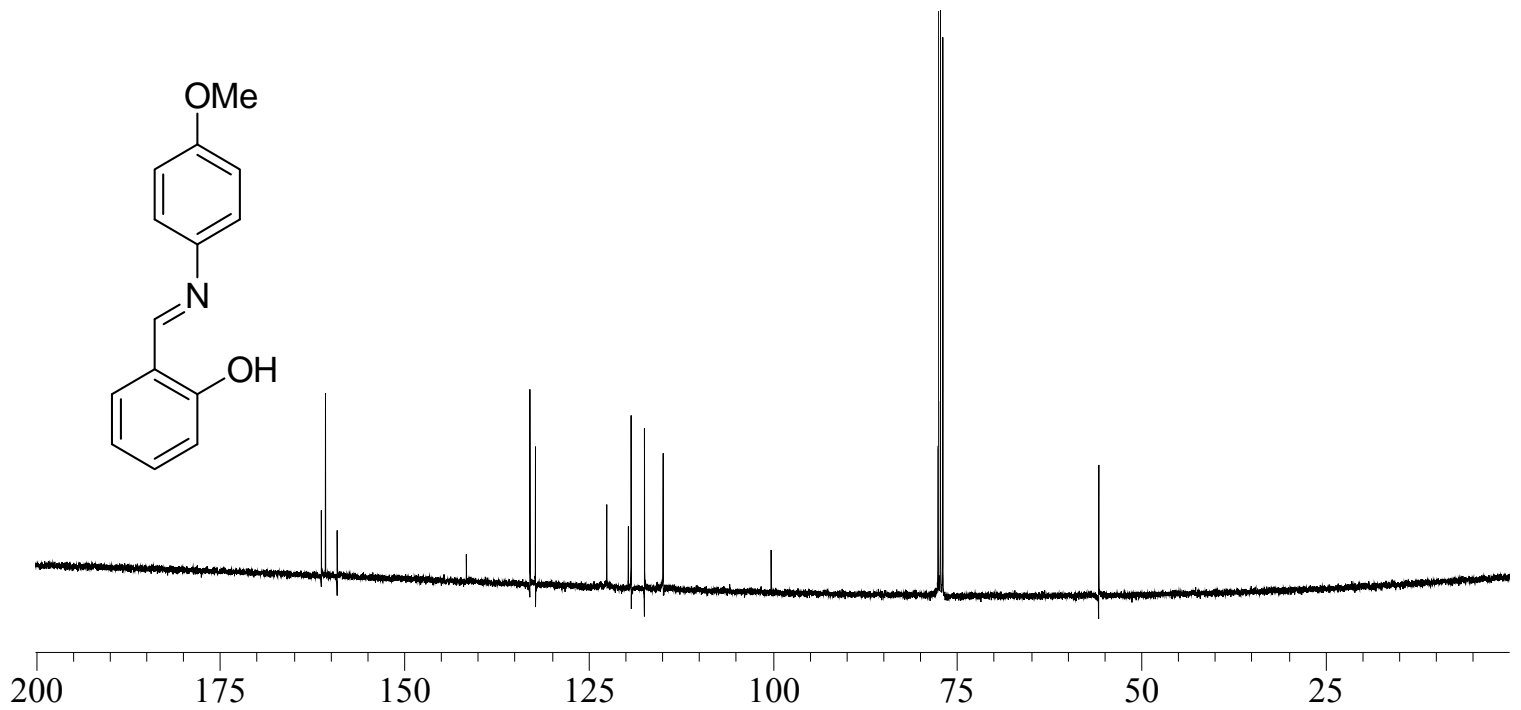

Figure S3. ${ }^{13} \mathrm{C}$ NMR spectrum of $N$-salicylidene- $p$-anisidine, 1 , in $\mathrm{CDCl}_{3}(100.6 \mathrm{MHz})$.

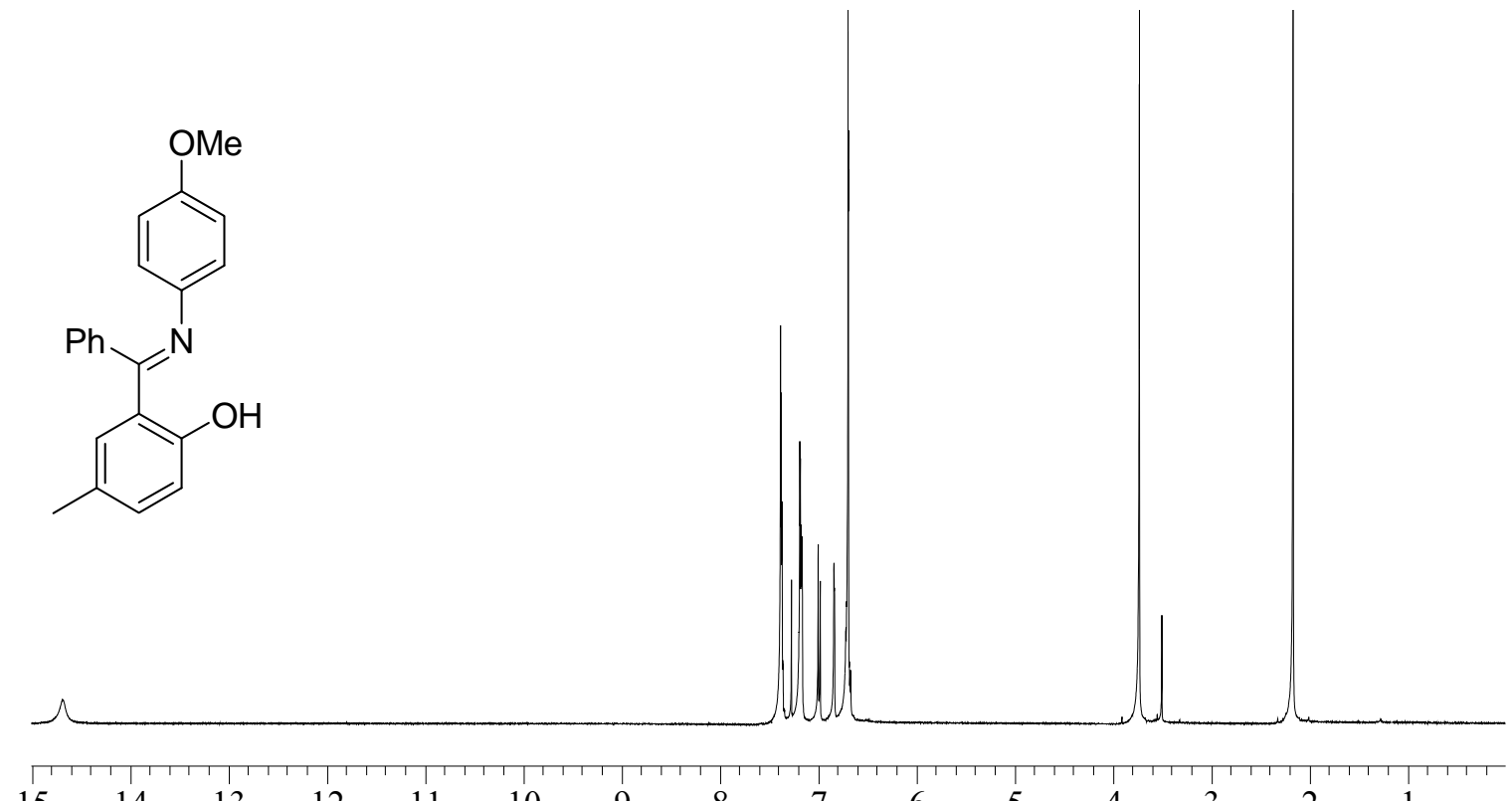

$\begin{array}{lllllllllllllll}15 & 14 & 13 & 12 & 11 & 10 & 9 & 8 & 7 & 6 & 5 & 4 & 3 & 2 & 1\end{array}$

Figure S4. ${ }^{1} \mathrm{H}$ NMR spectrum of $p$-anisidine ketimine, 2 , in $\mathrm{CDCl}_{3}(400 \mathrm{MHz})$. 


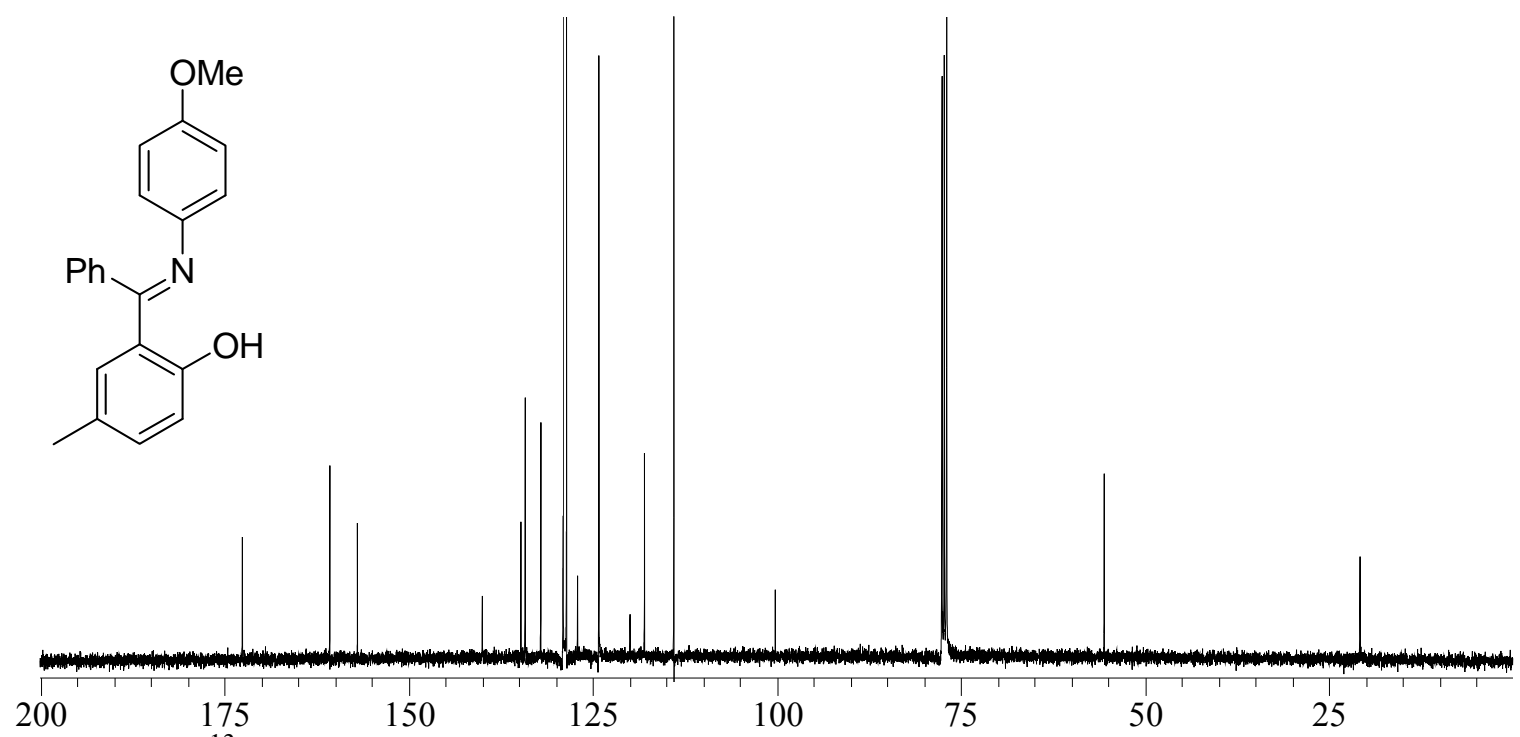

Figure S5. ${ }^{13} \mathrm{C}$ NMR spectrum of $p$-anisidine ketimine, 2, in $\mathrm{CDCl}_{3}(100.6 \mathrm{MHz})$.

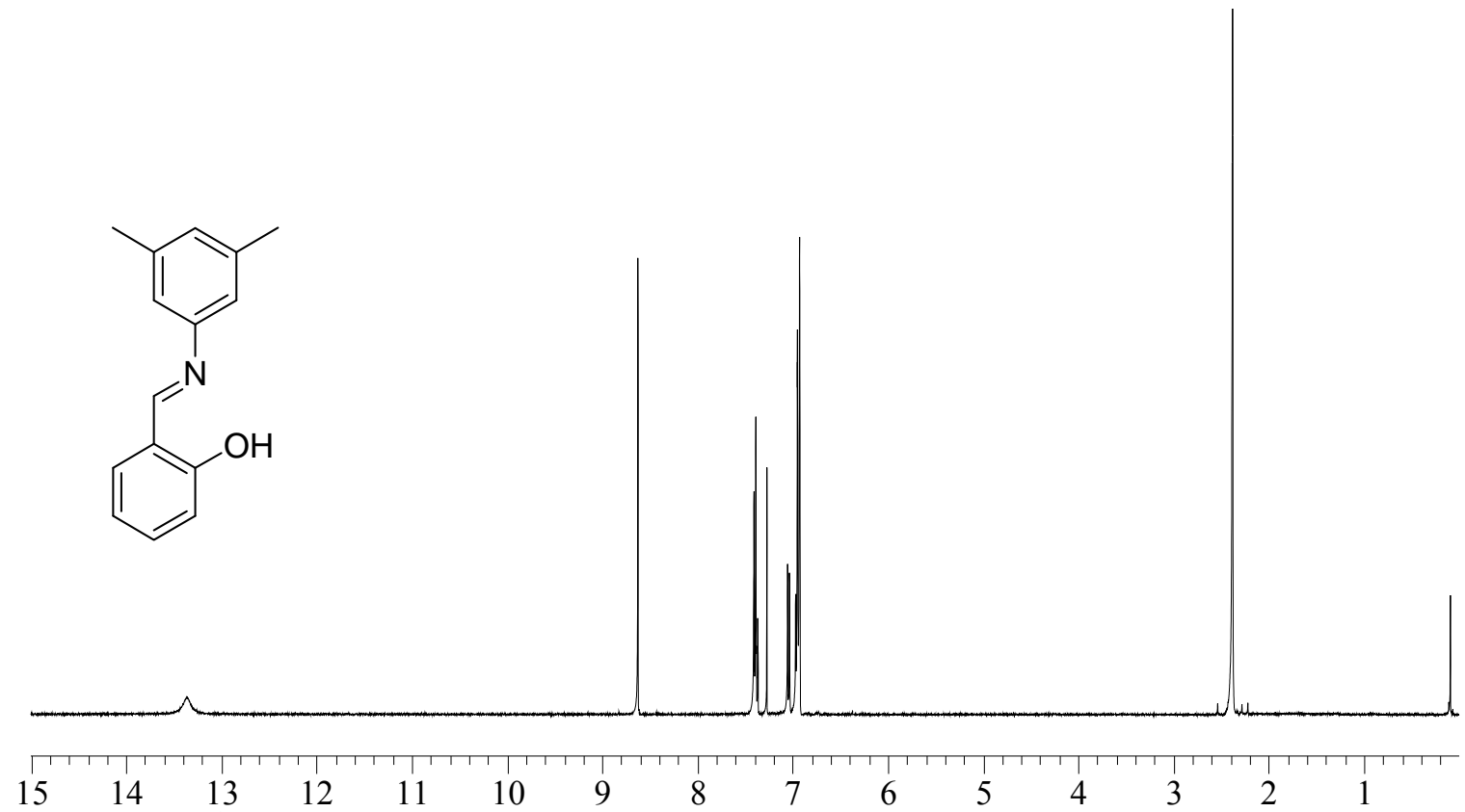

Figure S6. ${ }^{1} \mathrm{H}$ NMR spectrum of $N$-salicylidene-3,5-dimethylaniline, 3 , in $\mathrm{CDCl}_{3}$ (400 $\mathrm{MHz})$. 


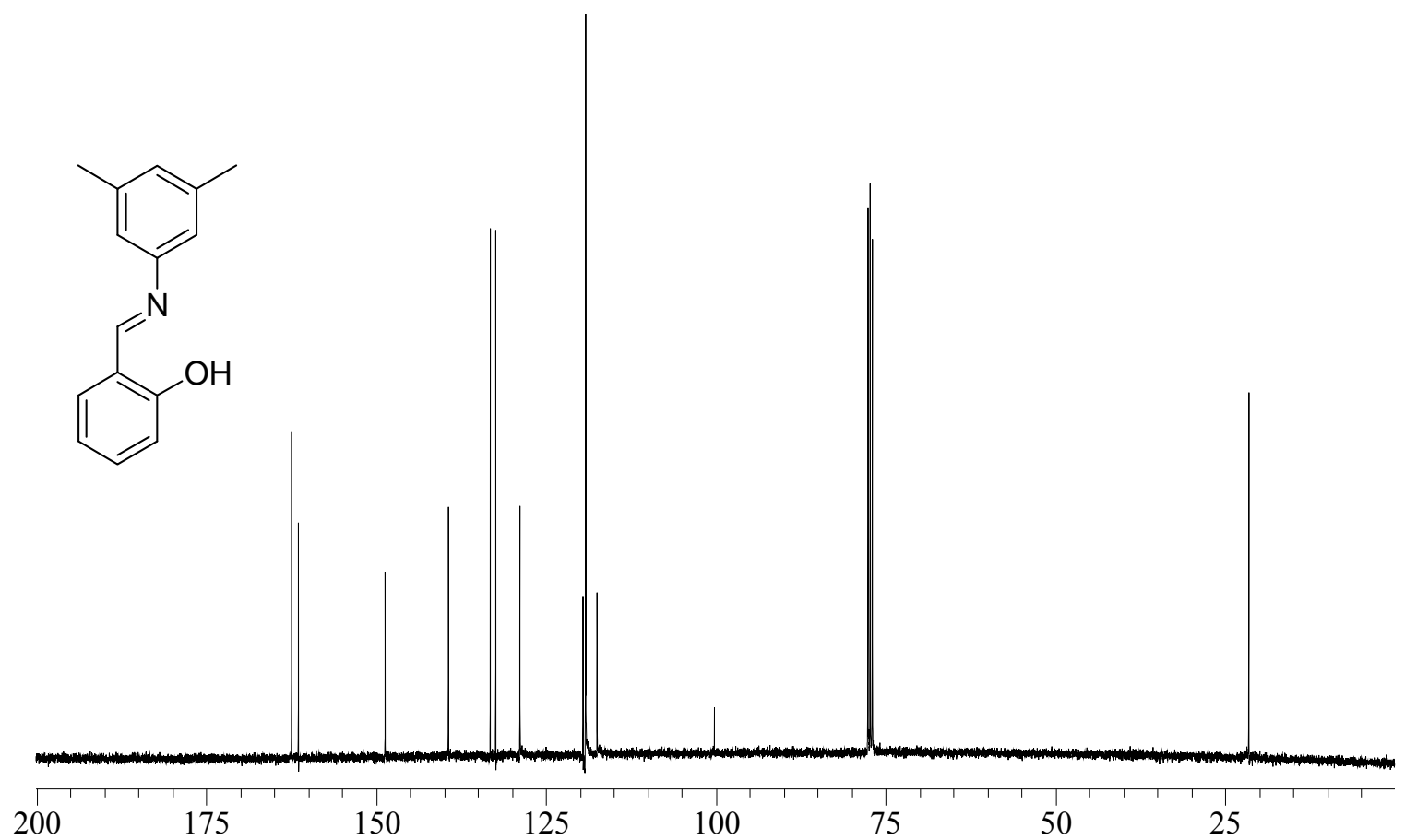

Figure S7. ${ }^{13} \mathrm{C}$ NMR spectrum of $N$-salicylidene-3,5-dimethylaniline, 3 , in $\mathrm{CDCl}_{3}(100.6$ $\mathrm{MHz})$.

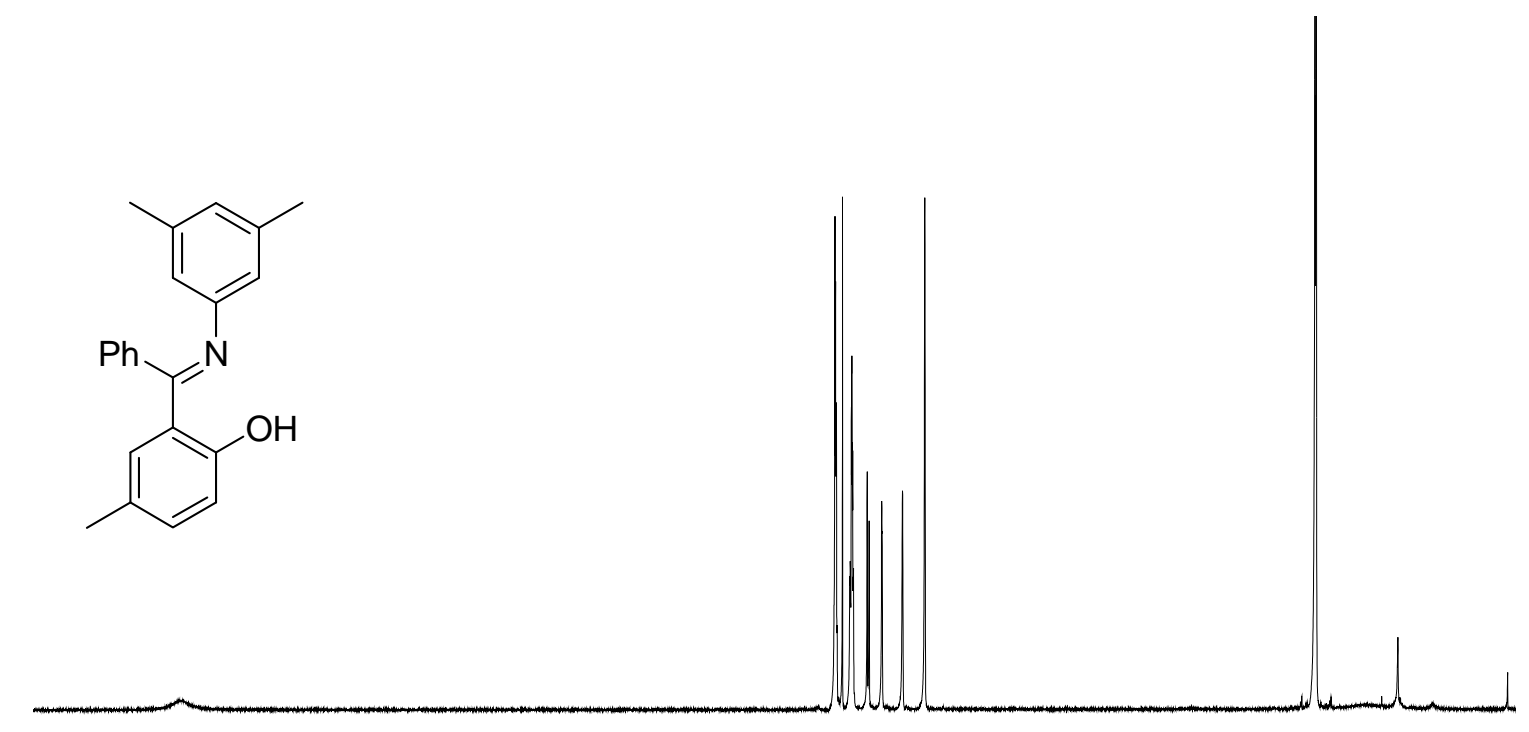

\begin{tabular}{llllllllllllllll|}
16 & 15 & 14 & 13 & 12 & 11 & 10 & 9 & 8 & 7 & 6 & 5 & 4 & 3 & 2 & 1
\end{tabular} Figure S8. ${ }^{1} \mathrm{H}$ NMR spectrum of 3,5-dimethylaniline ketimine, 4, in $\mathrm{CDCl}_{3}(400 \mathrm{MHz})$. 


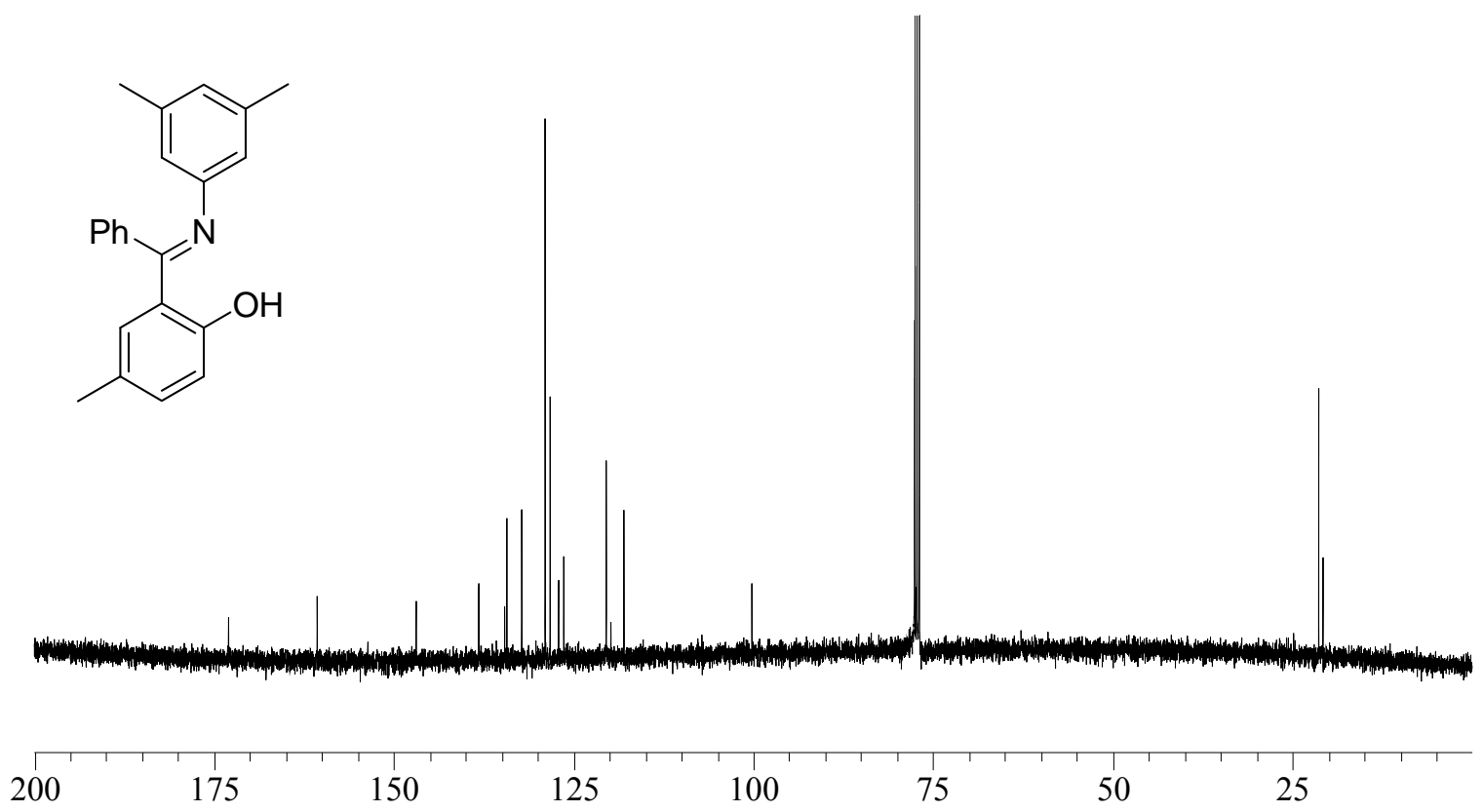

Figure S9. ${ }^{13} \mathrm{C}$ NMR spectrum of 3,5-dimethylaniline ketimine, 4, in $\mathrm{CDCl}_{3}(100.6$ $\mathrm{MHz}$ ).

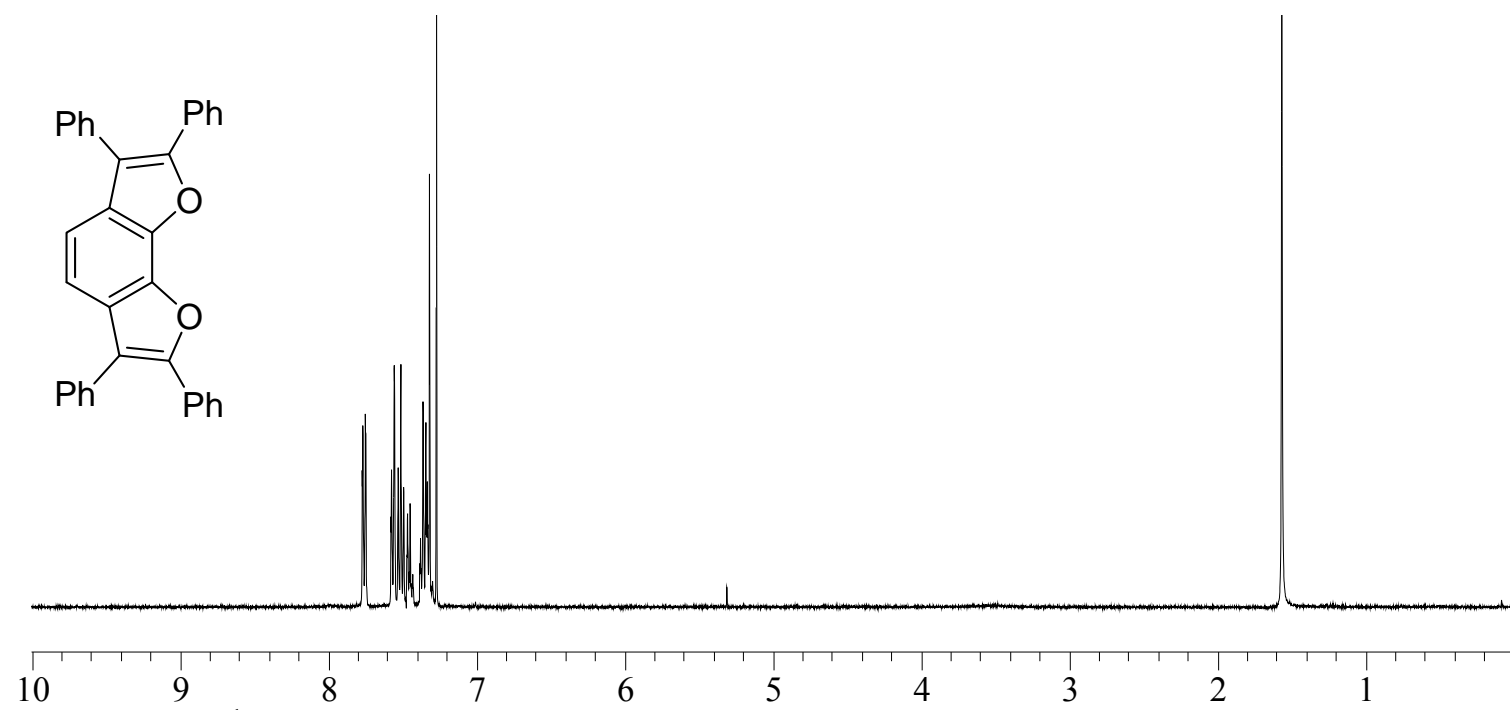

Figure S10. ${ }^{1} \mathrm{H}$ NMR spectrum of tetraphenyl-o-benzodifuran, 5, in $\mathrm{CDCl}_{3}(400 \mathrm{MHz})$. 


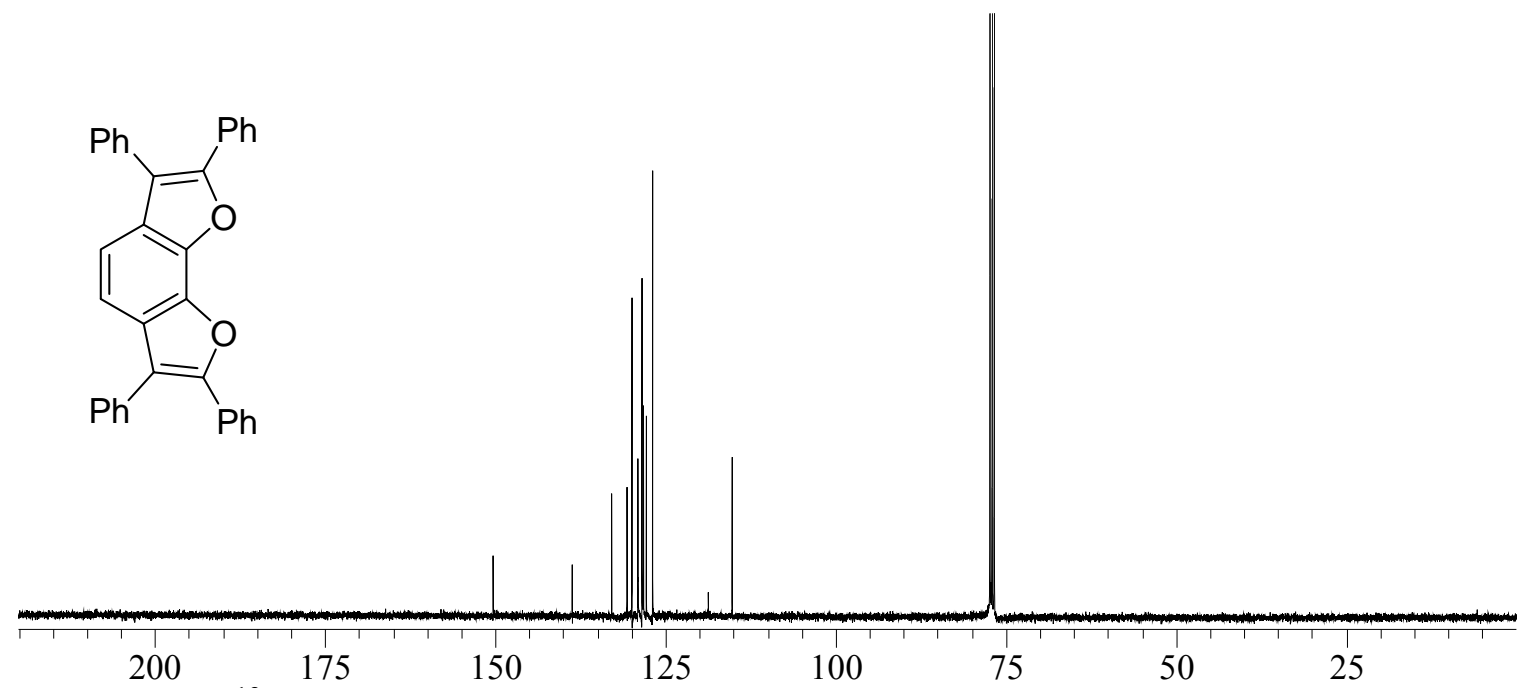

Figure S11. ${ }^{13} \mathrm{C}$ NMR spectrum of tetraphenyl-o-benzodifuran, 5, in $\mathrm{CDCl}_{3}(100.6 \mathrm{MHz})$.

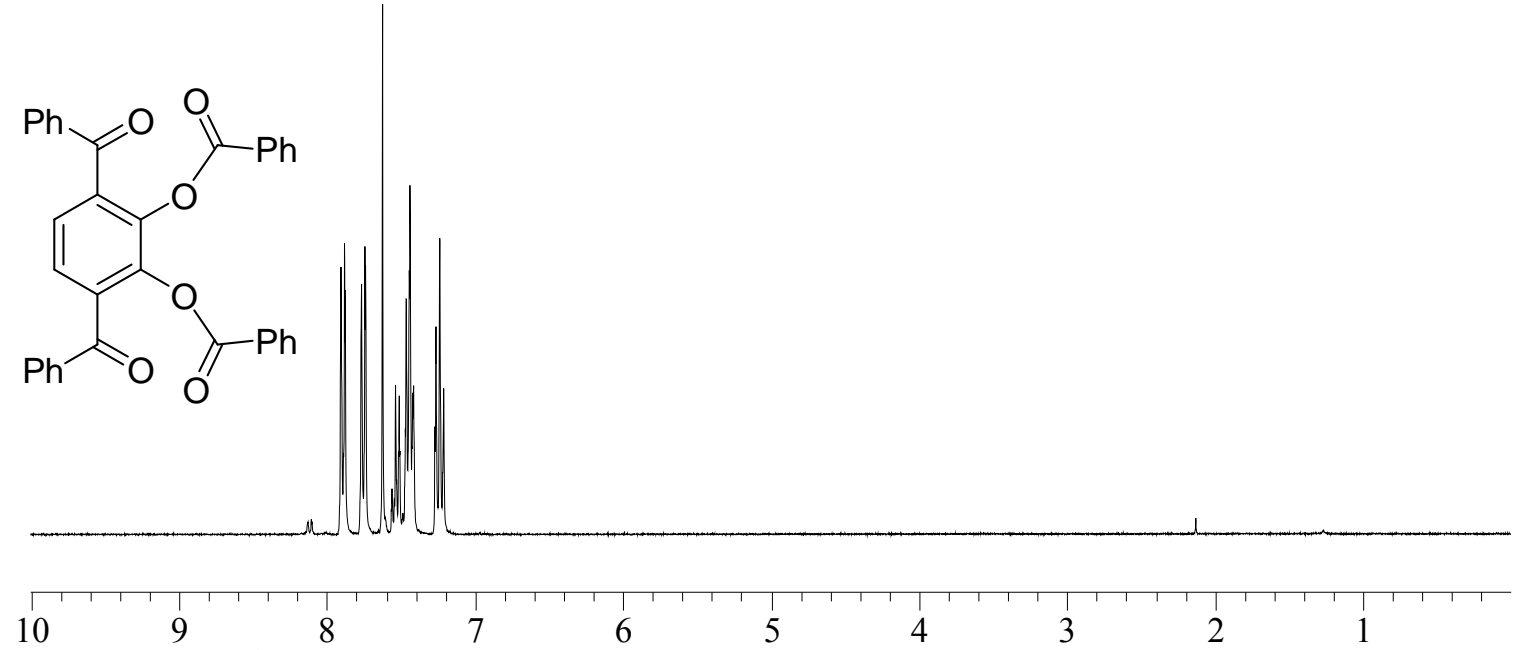

Figure S12. ${ }^{1} \mathrm{H}$ NMR spectrum of 2,3-bis(benzoyloxy)-1,4-dibenzoylbenzene, $5 \mathbf{b}$, in $\mathrm{CDCl}_{3}(300 \mathrm{MHz})$. 
<smiles>O=C(Oc1c(C(=O)c2ccccc2)ccc(C(=O)c2ccccc2)c1OC(=O)c1ccccc1)c1ccccc1</smiles>

Figure S13. ${ }^{13} \mathrm{C}$ NMR spectrum of 2,3-bis(benzoyloxy)-1,4-dibenzoylbenzene, $\mathbf{5 b}$, in $\mathrm{CDCl}_{3}(100.6 \mathrm{MHz})$.

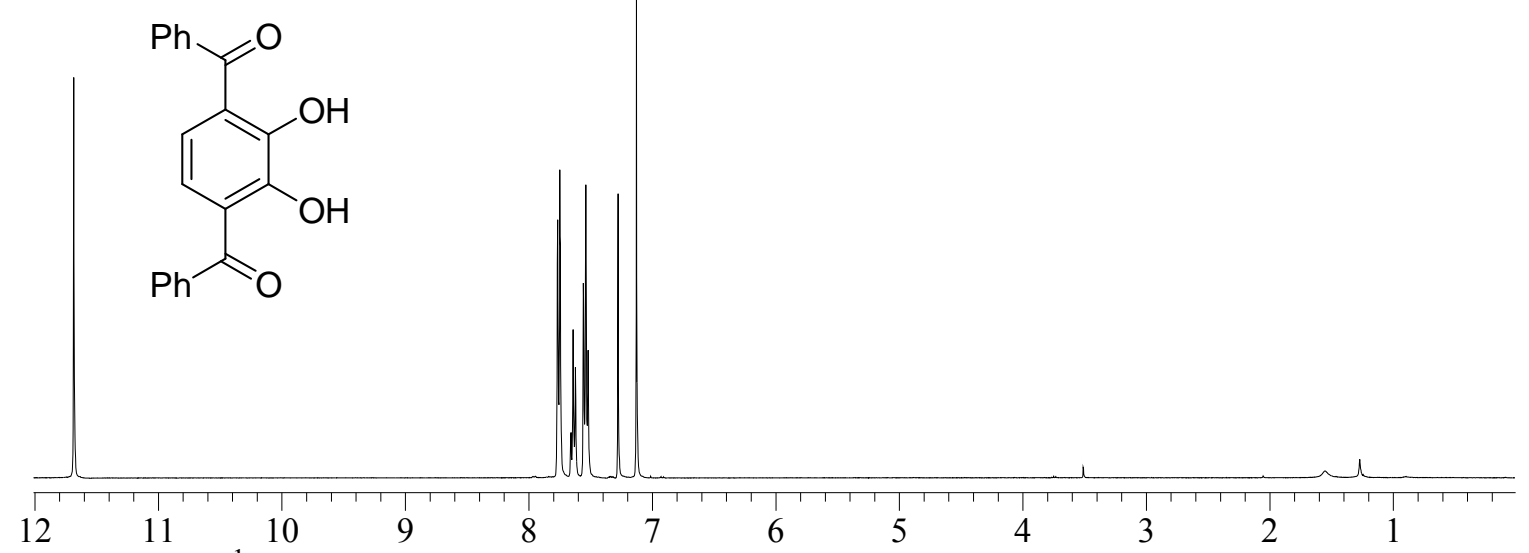

Figure S14. ${ }^{1} \mathrm{H}$ NMR spectrum of 3,6-dibenzoylcatechol, 6, in $\mathrm{CDCl}_{3}(400 \mathrm{MHz})$.

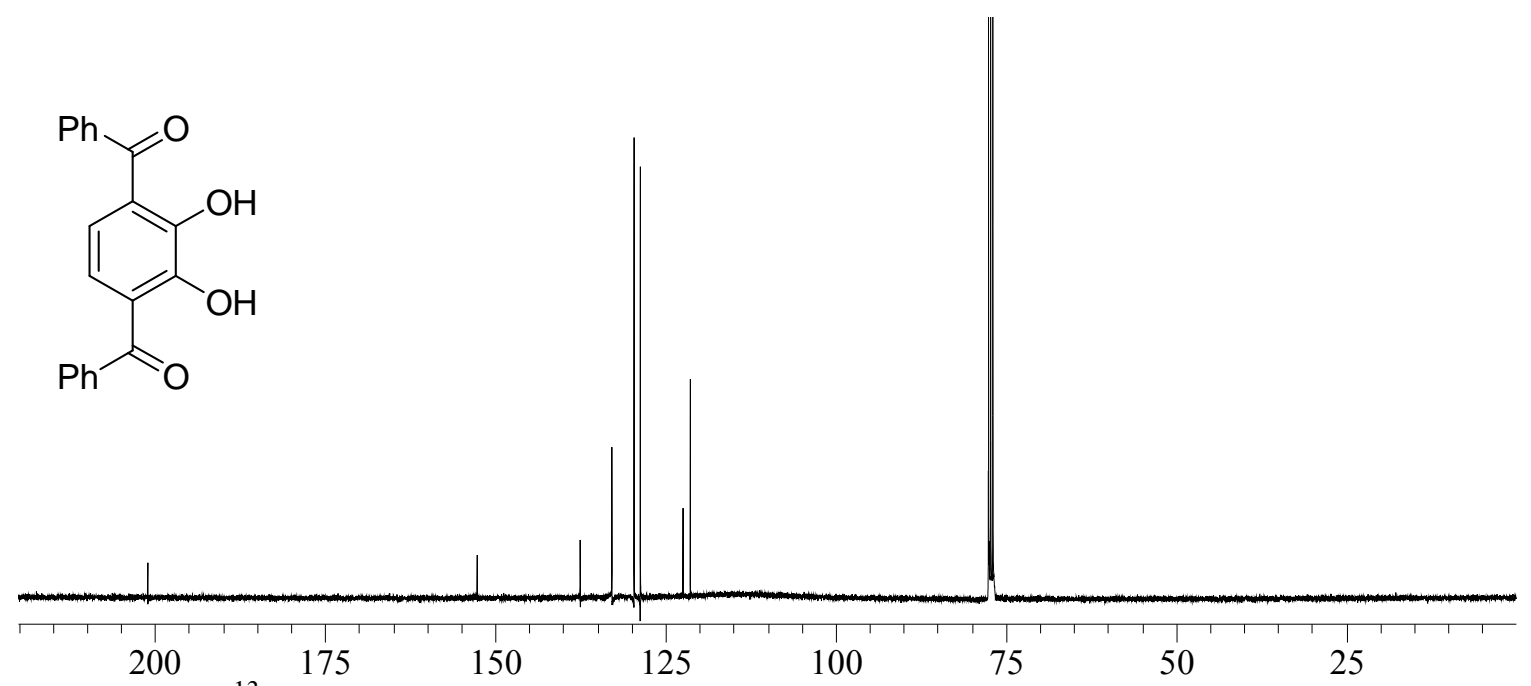

Figure S15. ${ }^{13} \mathrm{C}$ NMR spectrum of 3,6-dibenzoylcatechol, 6, in $\mathrm{CDCl}_{3}(100.6 \mathrm{MHz})$. 


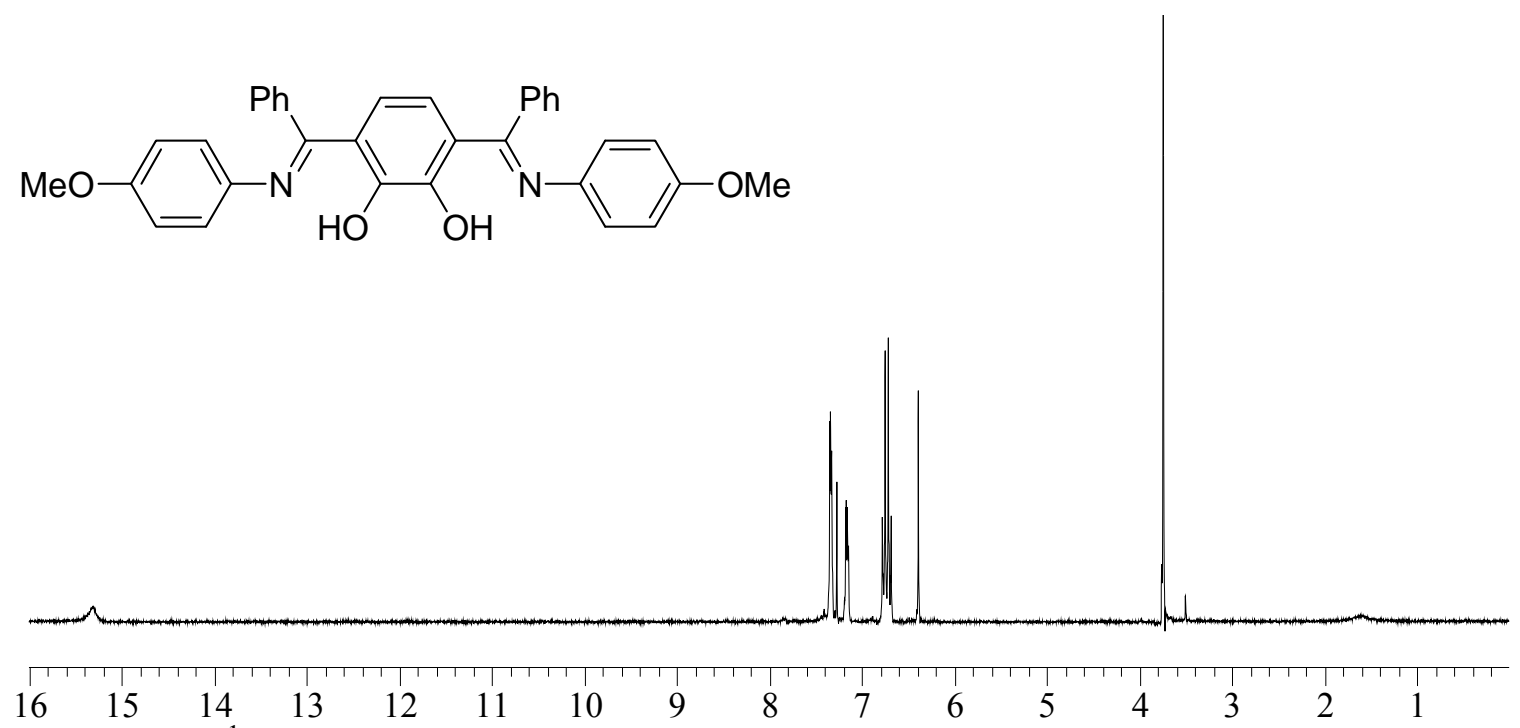

Figure S16. ${ }^{1} \mathrm{H}$ NMR spectrum of diketimine model compound, 8, in $\mathrm{CDCl}_{3}(400 \mathrm{MHz})$.

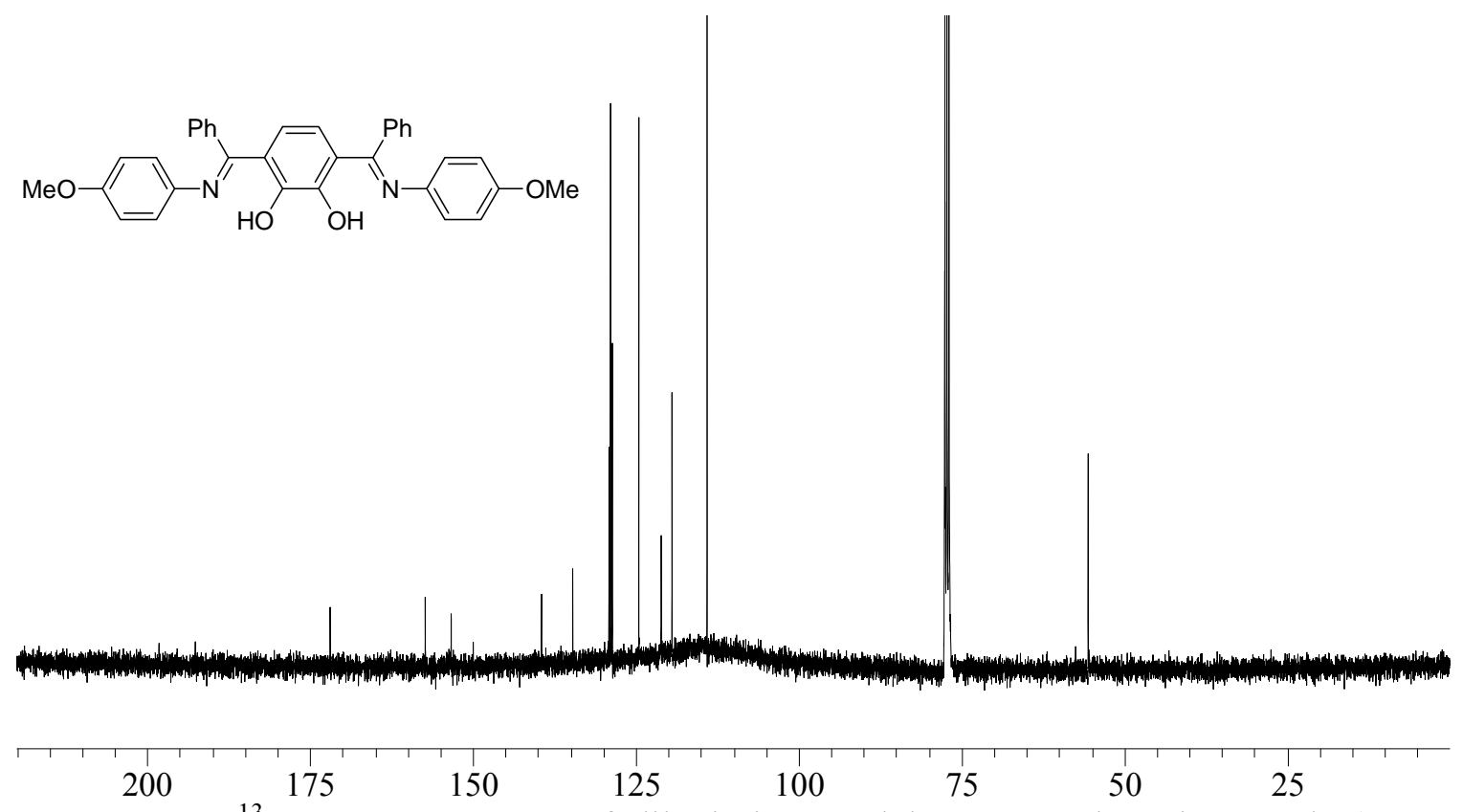

Figure S17. ${ }^{13} \mathrm{C}$ NMR spectrum of diketimine model compound, 8, in $\mathrm{CDCl}_{3}(100.6$ $\mathrm{MHz})$. 

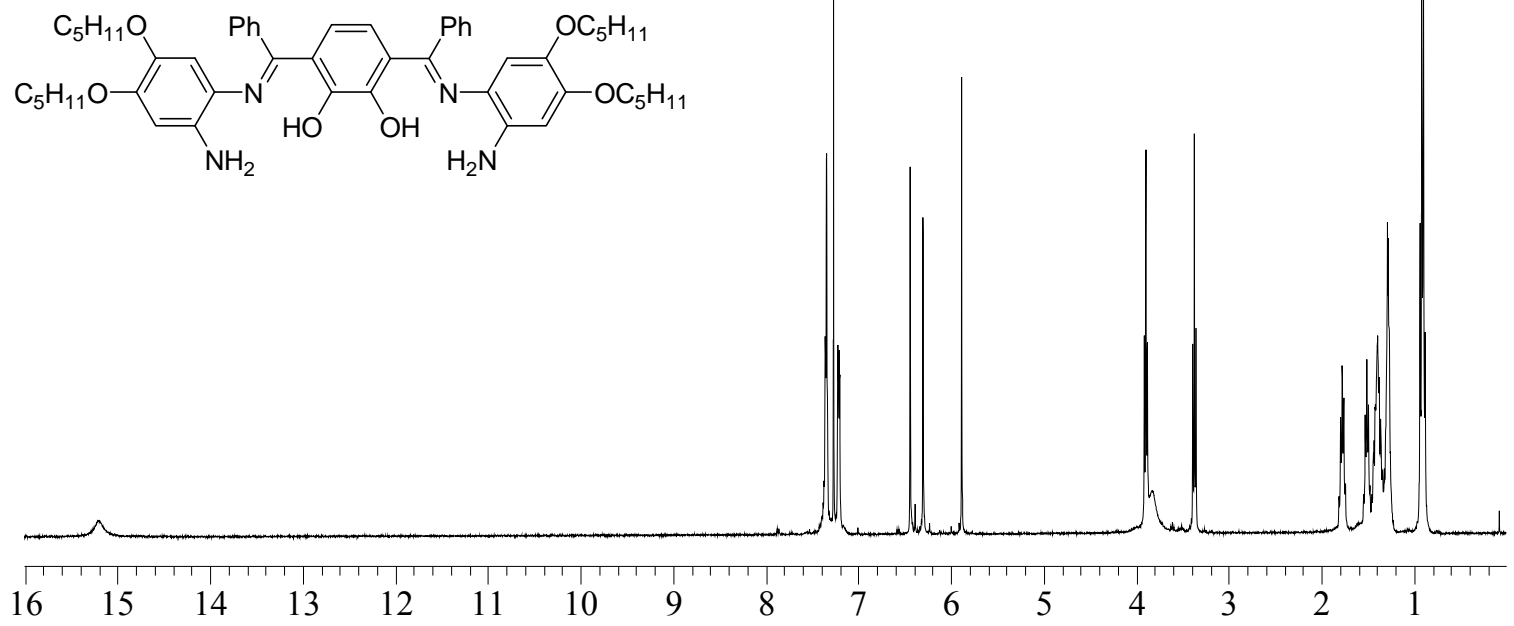

Figure S18. ${ }^{1} \mathrm{H}$ NMR spectrum of compound 10 in $\mathrm{CDCl}_{3}(400 \mathrm{MHz})$.

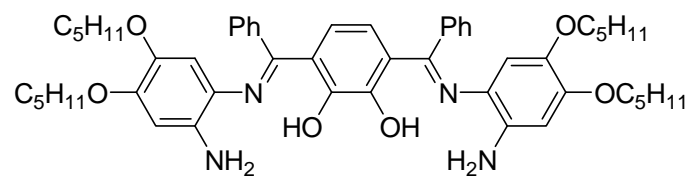
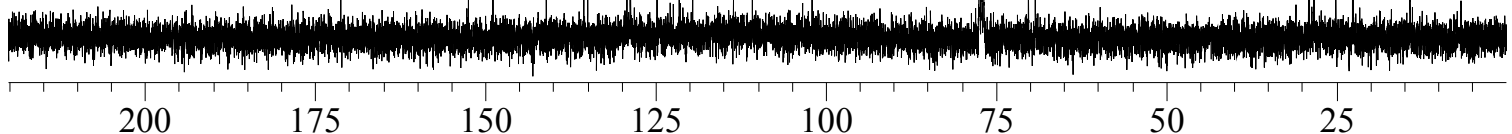

175

150

125

100

75

50

25

Figure S19. ${ }^{13} \mathrm{C}$ NMR spectrum of compound 10 in $\mathrm{CDCl}_{3}(100.6 \mathrm{MHz})$.

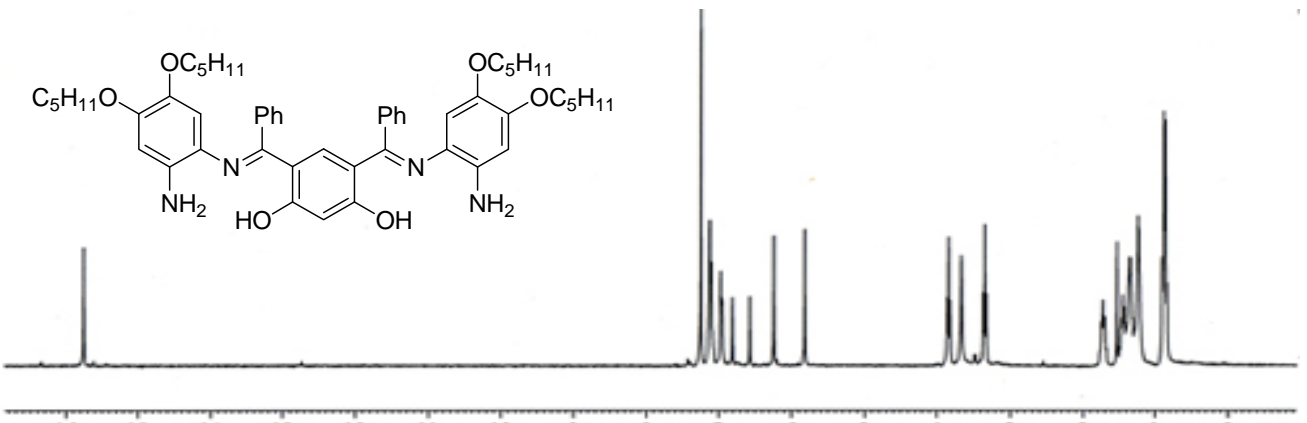

Figure S20. ${ }^{1} \mathrm{H}$ NMR spectrum of compound 11a in $\mathrm{CDCl}_{3}(400 \mathrm{MHz})$. 


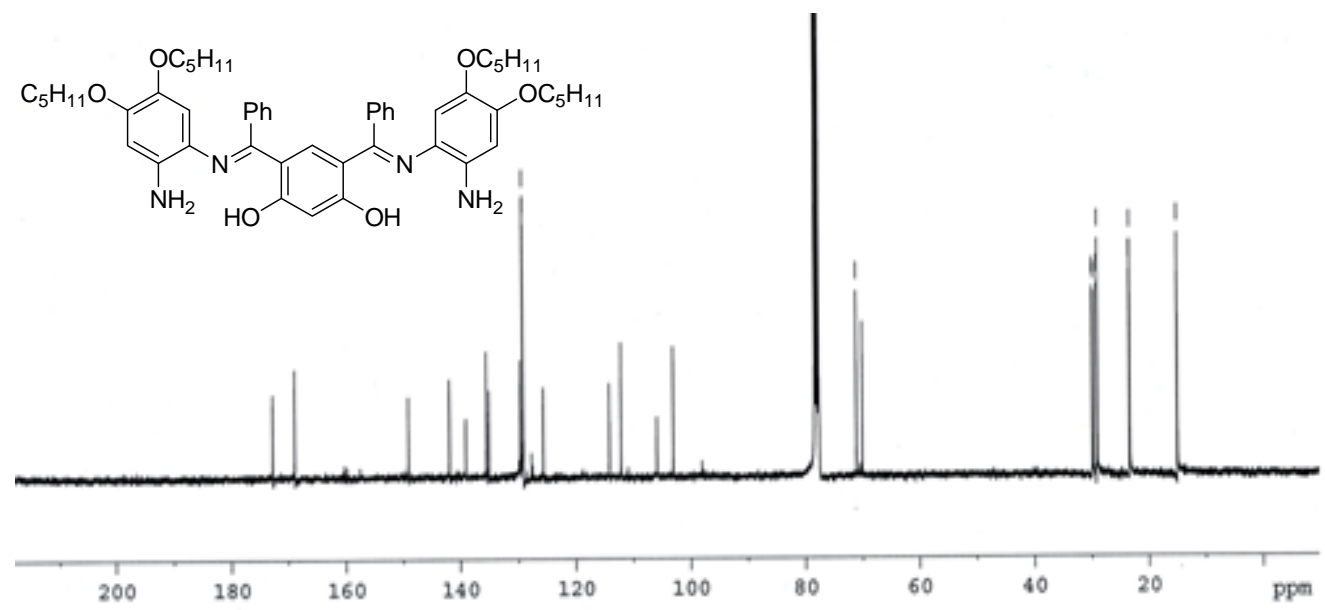

Figure S21. ${ }^{13} \mathrm{C}$ NMR spectrum of compound 11a in $\mathrm{CDCl}_{3}(100.6 \mathrm{MHz})$.

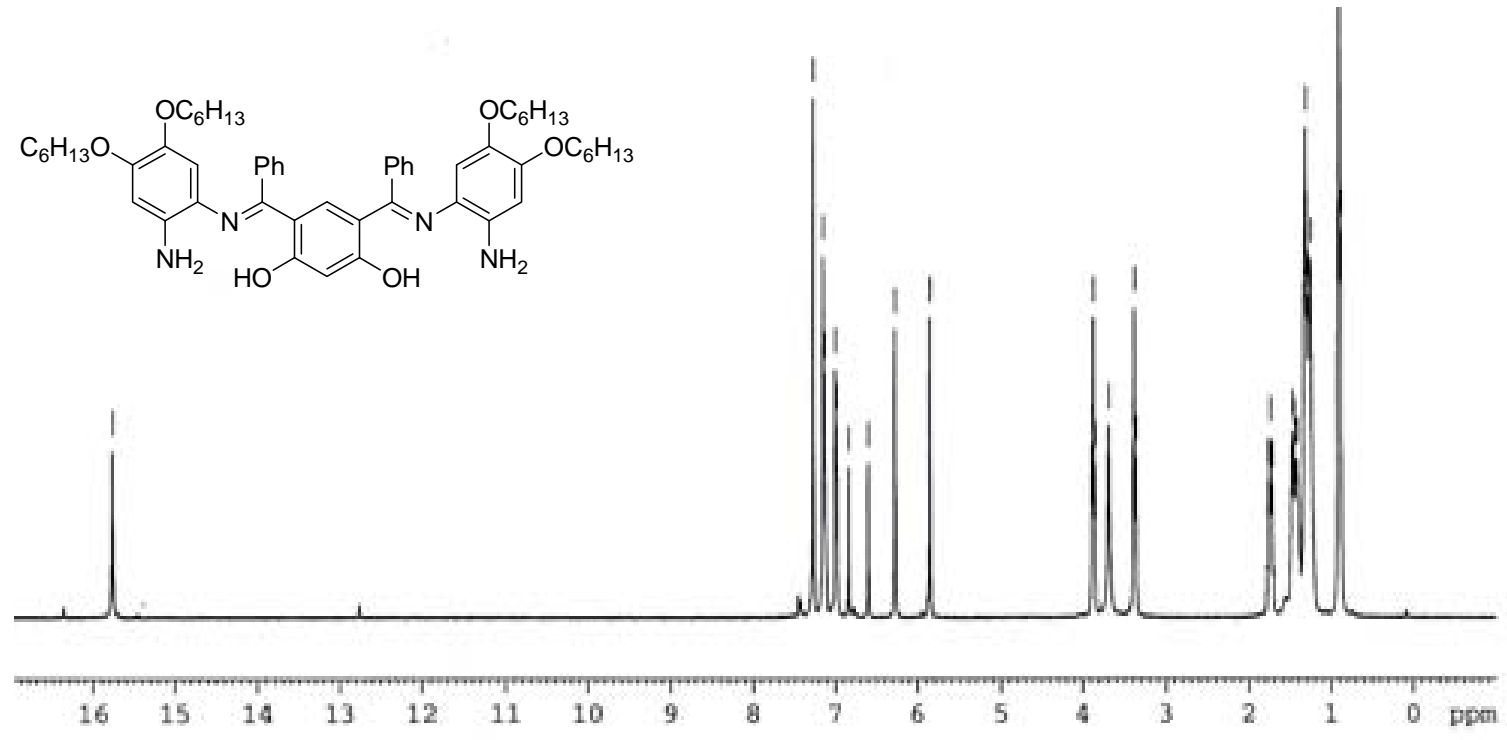

Figure S22. ${ }^{1} \mathrm{H}$ NMR spectrum of compound $\mathbf{1 1 b}$ in $\mathrm{CDCl}_{3}(400 \mathrm{MHz})$. 


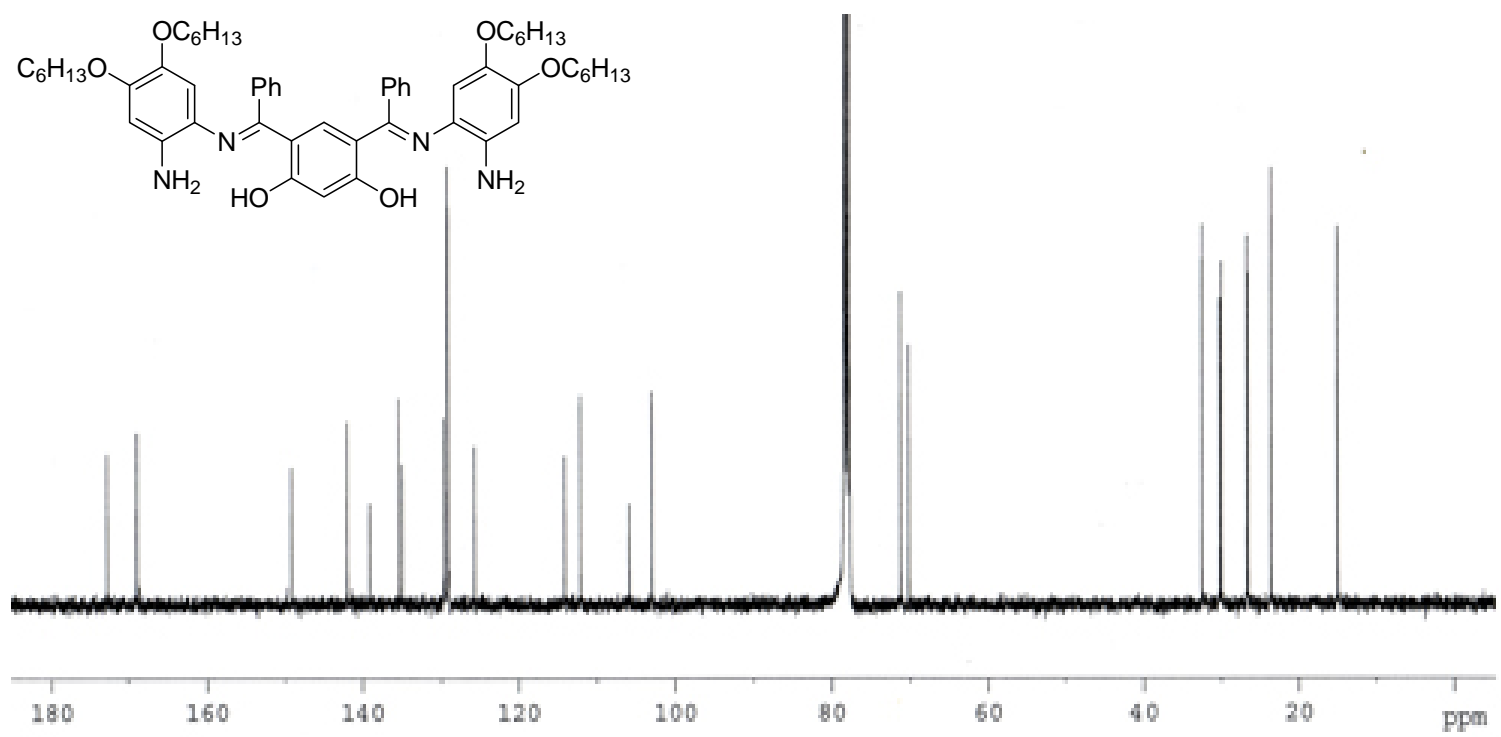

Figure S23. ${ }^{13} \mathrm{C}$ NMR spectrum of compound $\mathbf{1 1 b}$ in $\mathrm{CDCl}_{3}(100.6 \mathrm{MHz})$.

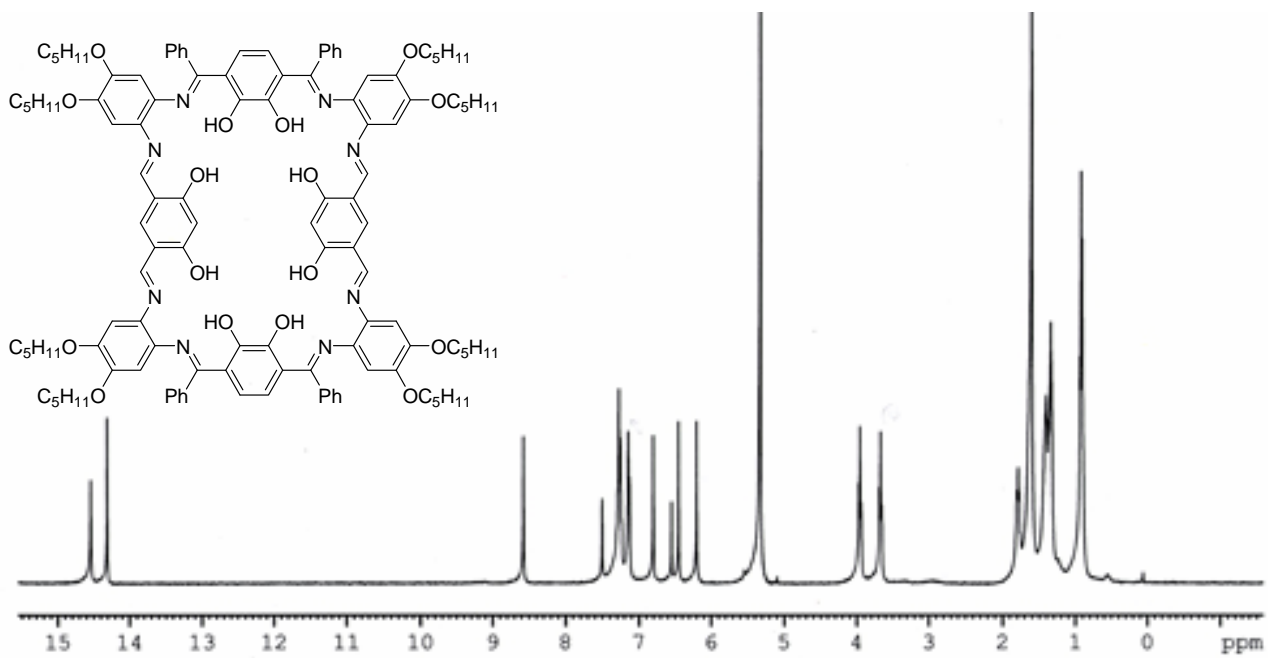

Figure S24. ${ }^{1} \mathrm{H}$ NMR spectrum of macrocycle 12 in $\mathrm{CD}_{2} \mathrm{Cl}_{2}(400 \mathrm{MHz})$.

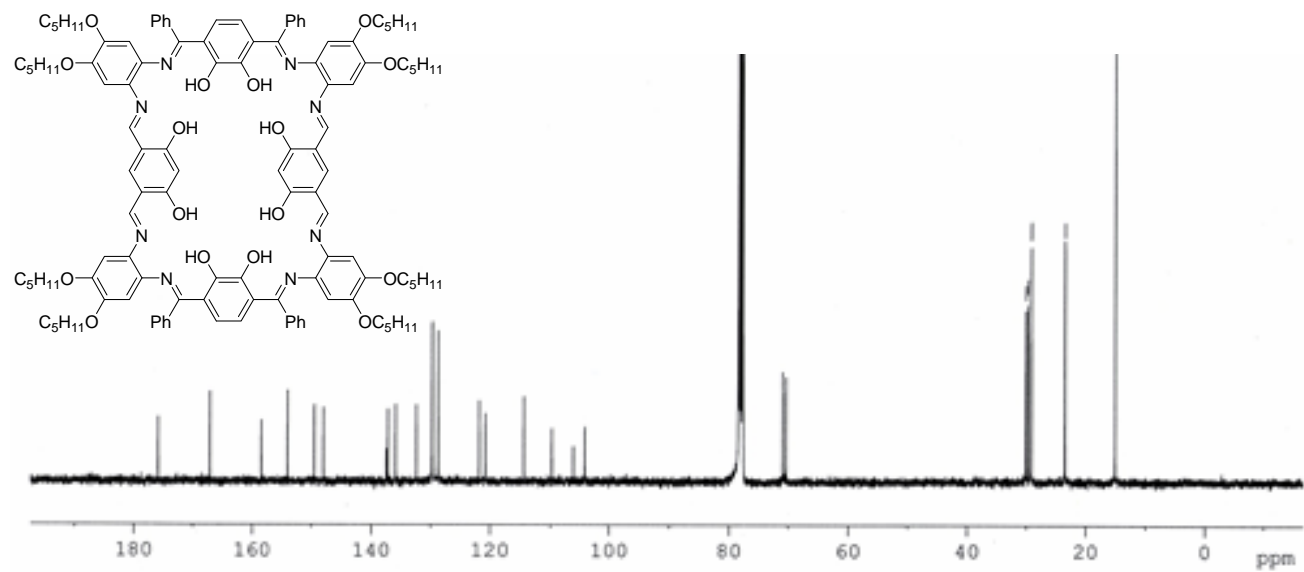

Figure S25. ${ }^{13} \mathrm{C}$ NMR spectrum of macrocycle 12 in $\mathrm{CDCl}_{3}(100.6 \mathrm{MHz})$. 


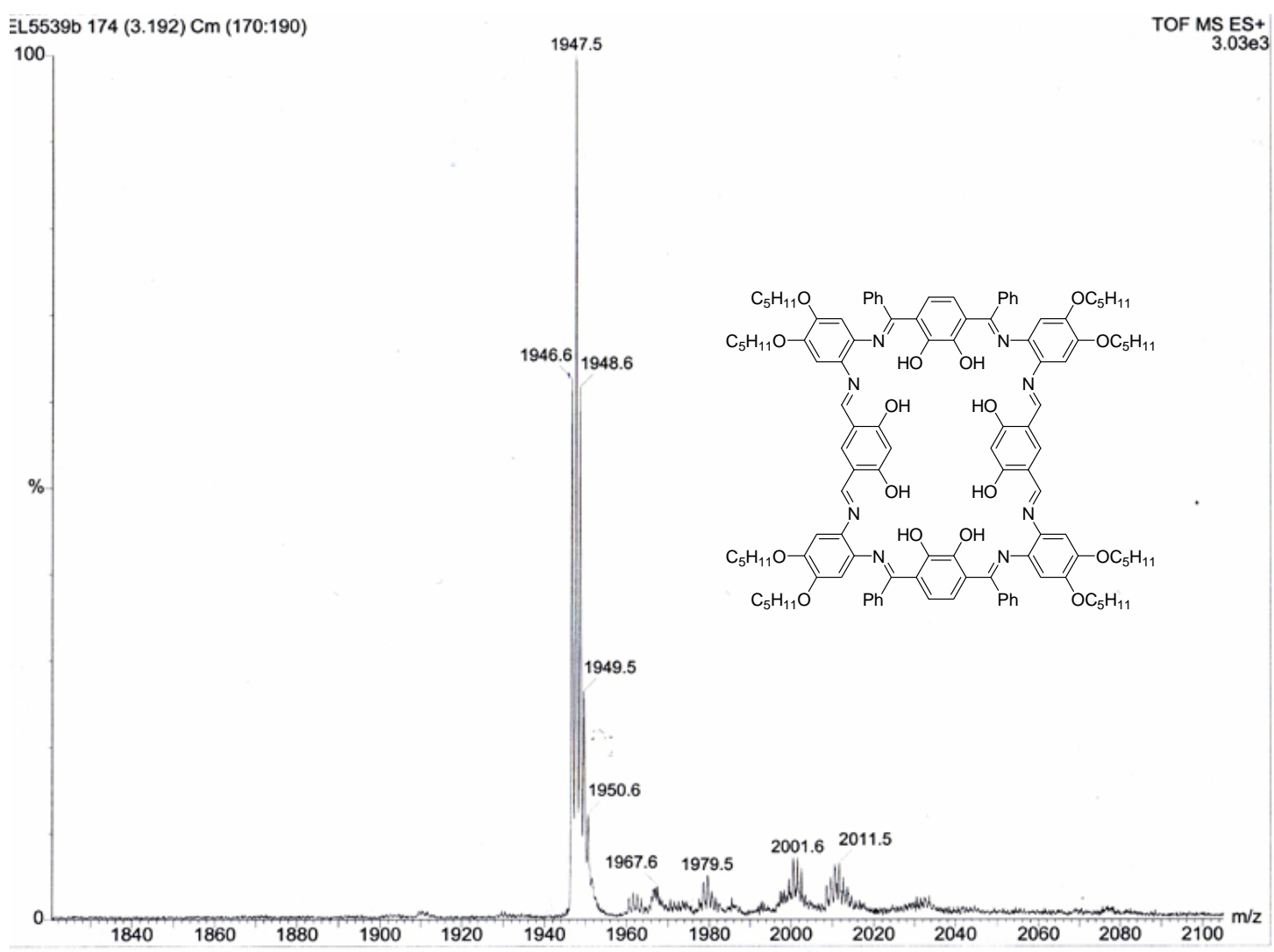

Figure S26. ESI mass spectrum of macrocycle 12.

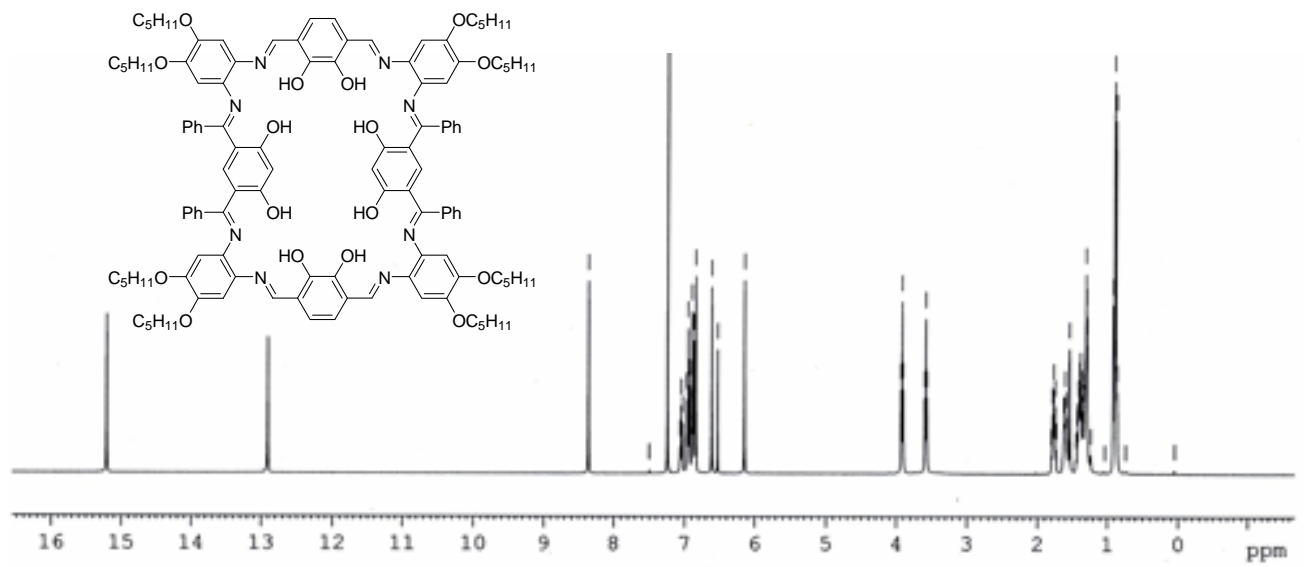

Figure S27. ${ }^{1} \mathrm{H}$ NMR spectrum of macrocycle 13 in $\mathrm{CDCl}_{3}(400 \mathrm{MHz})$. 


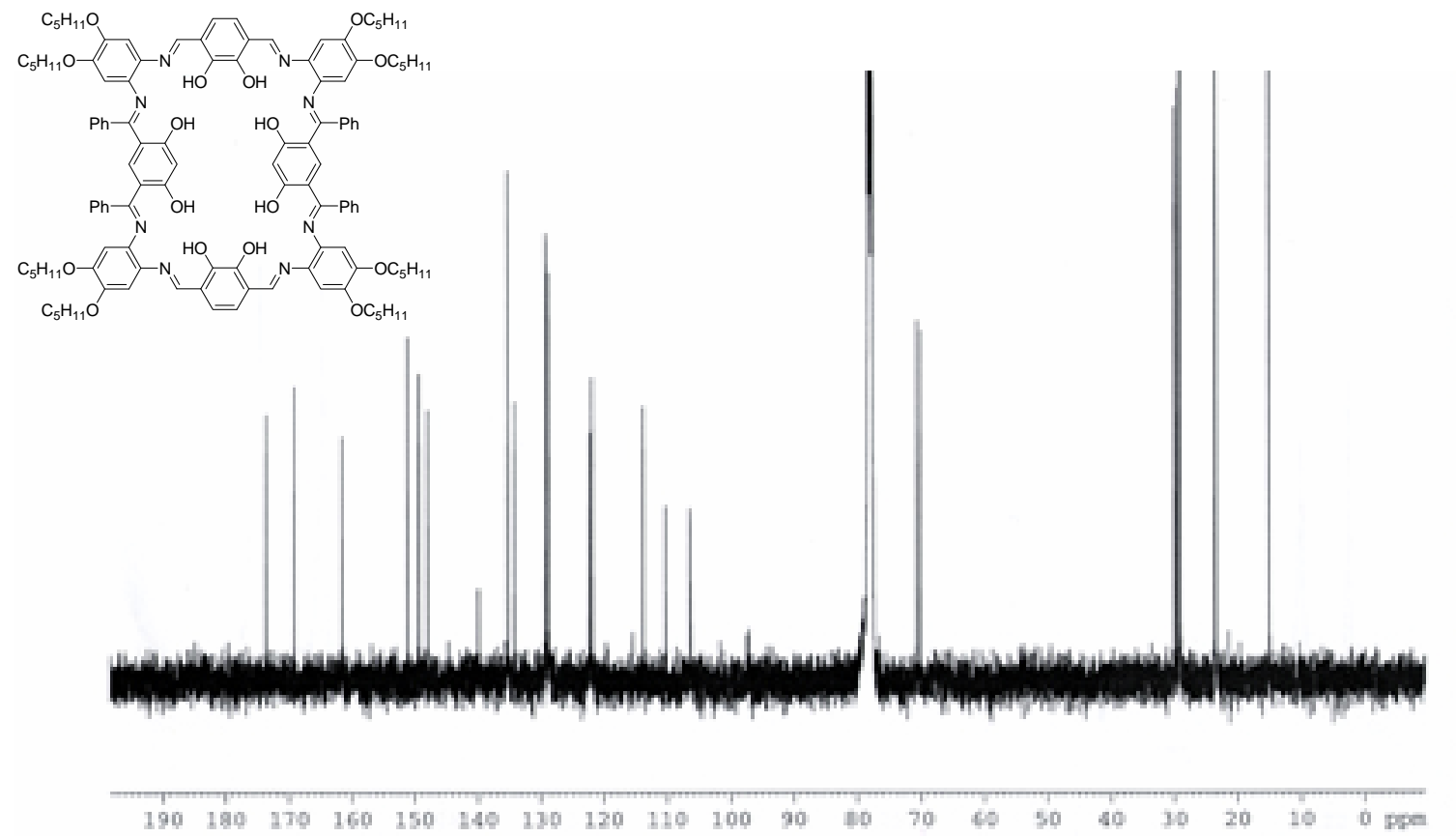

Figure S28. ${ }^{13} \mathrm{C}$ NMR spectrum of macrocycle 13 in $\mathrm{CDCl}_{3}(100.6 \mathrm{MHz})$.

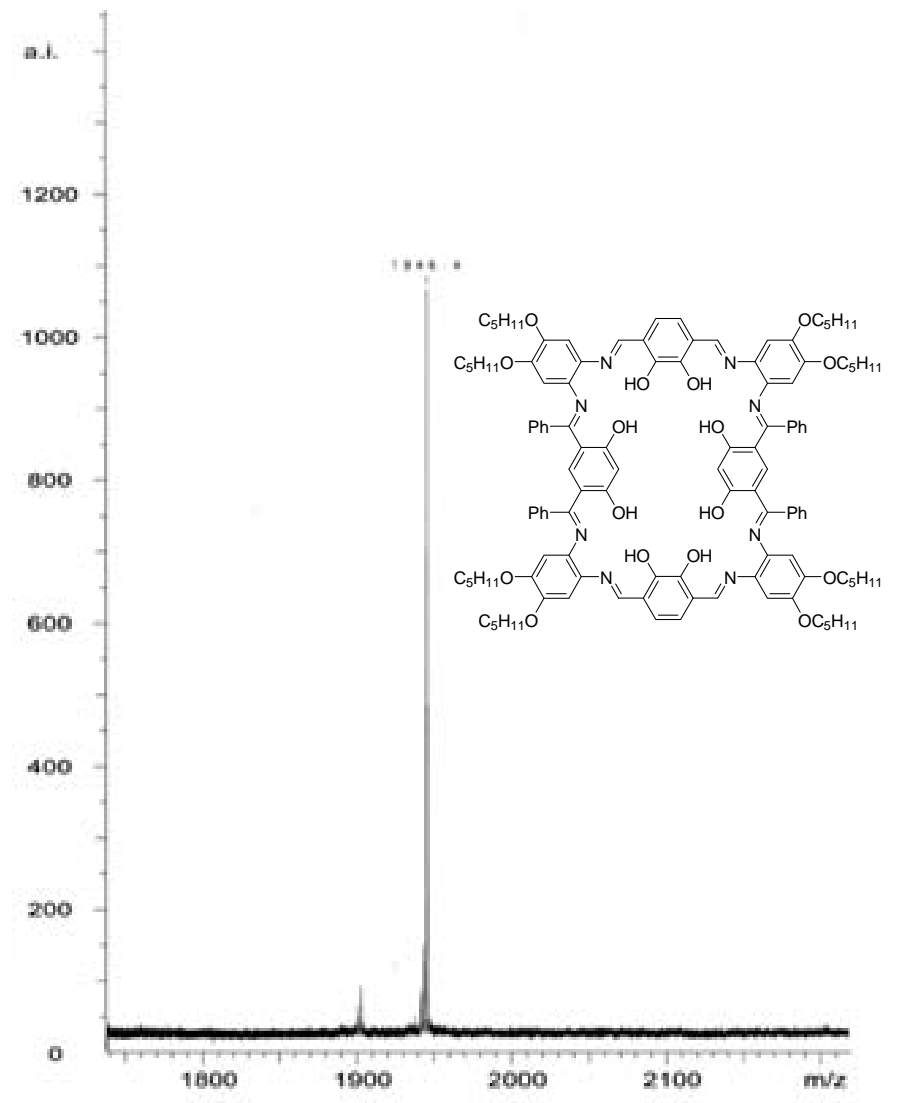

Figure S29. MALDI-TOF mass spectrum of macrocycle $\mathbf{1 3}$ (dithranol matrix). 


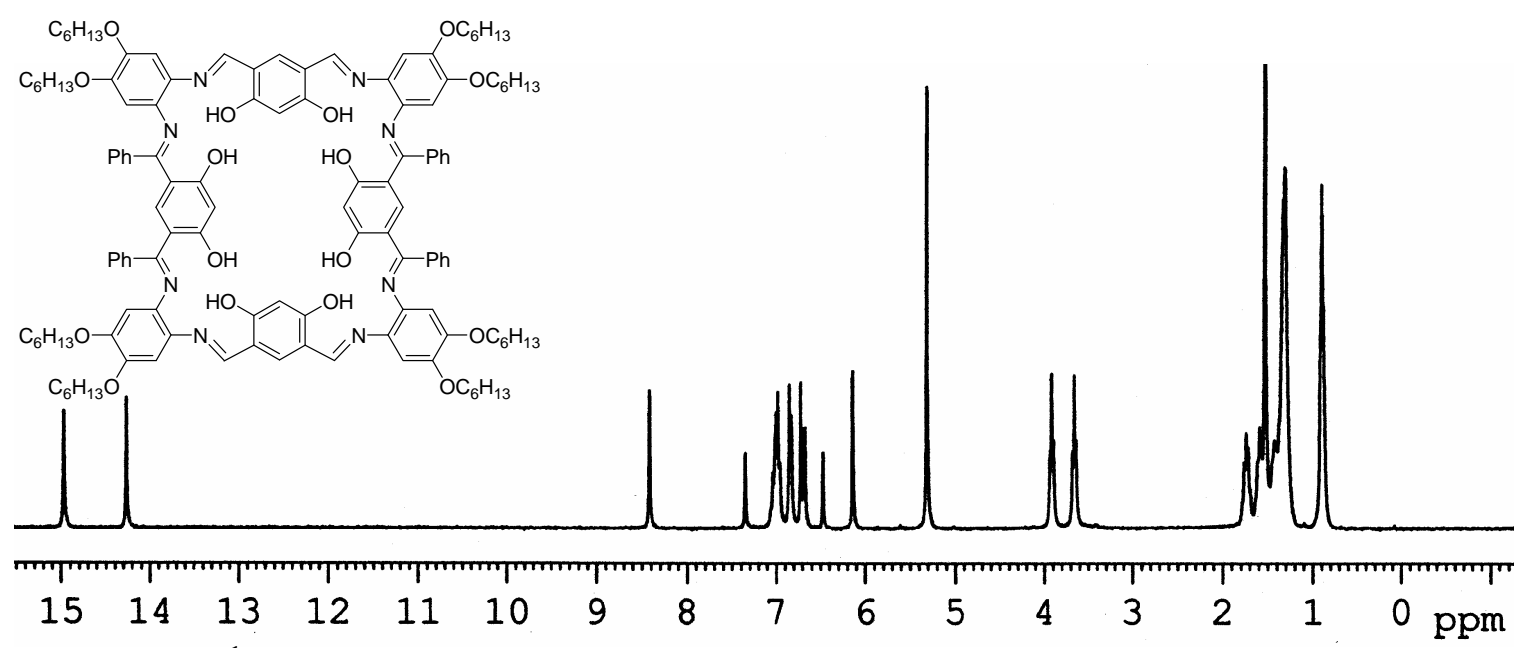

Figure S30. ${ }^{1} \mathrm{H}$ NMR spectrum of macrocycle 14 in $\mathrm{CD}_{2} \mathrm{Cl}_{2}(300 \mathrm{MHz})$.

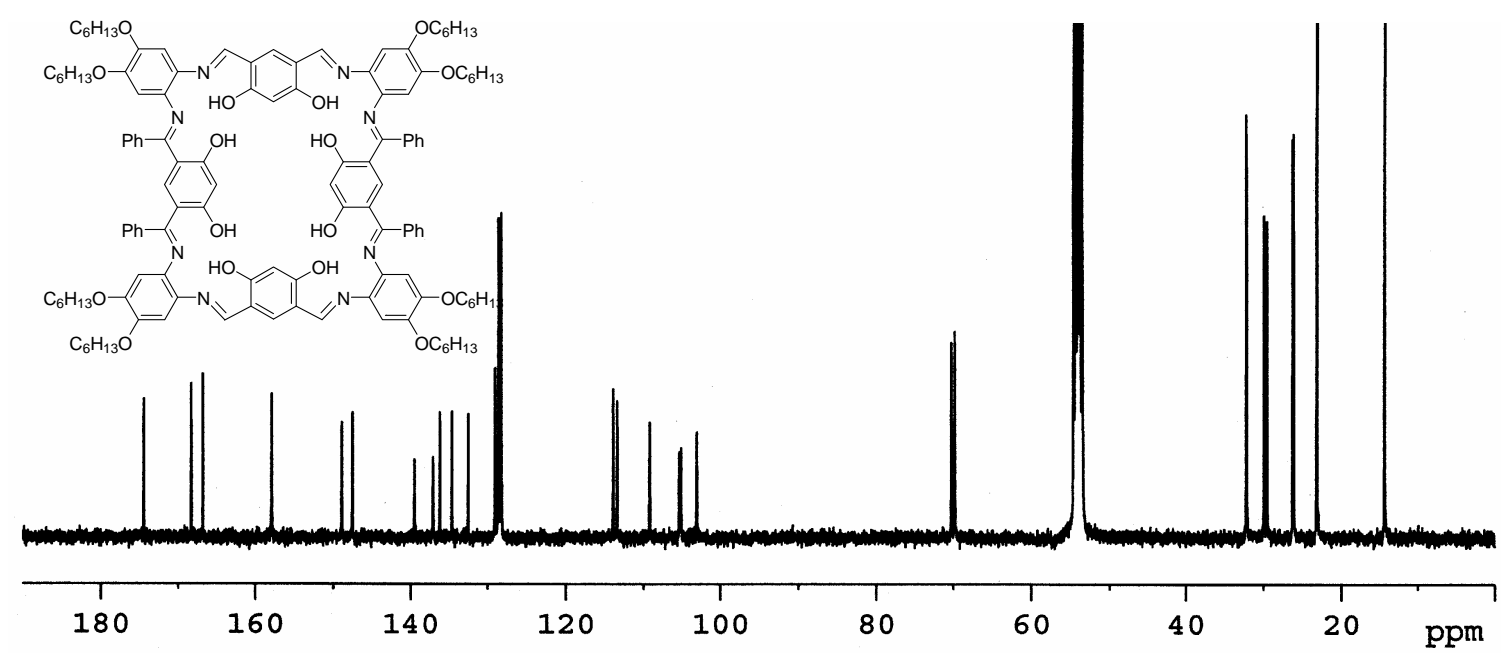

Figure S31. ${ }^{13} \mathrm{C}$ NMR spectrum of macrocycle 14 in $\mathrm{CD}_{2} \mathrm{Cl}_{2}(100.6 \mathrm{MHz})$. 


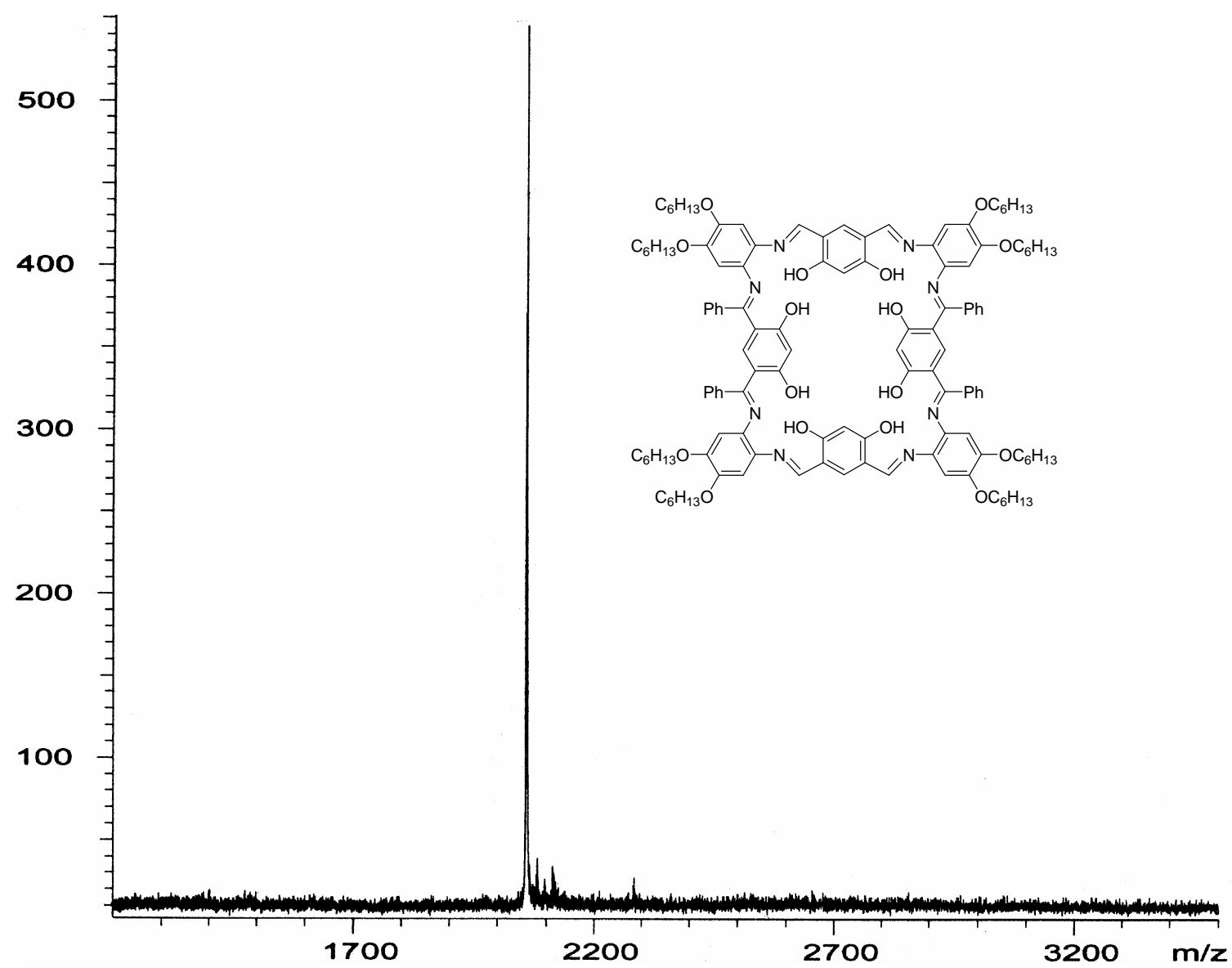

Figure S32. MALDI-TOF mass spectrum of macrocycle 14 (dithranol matrix). 


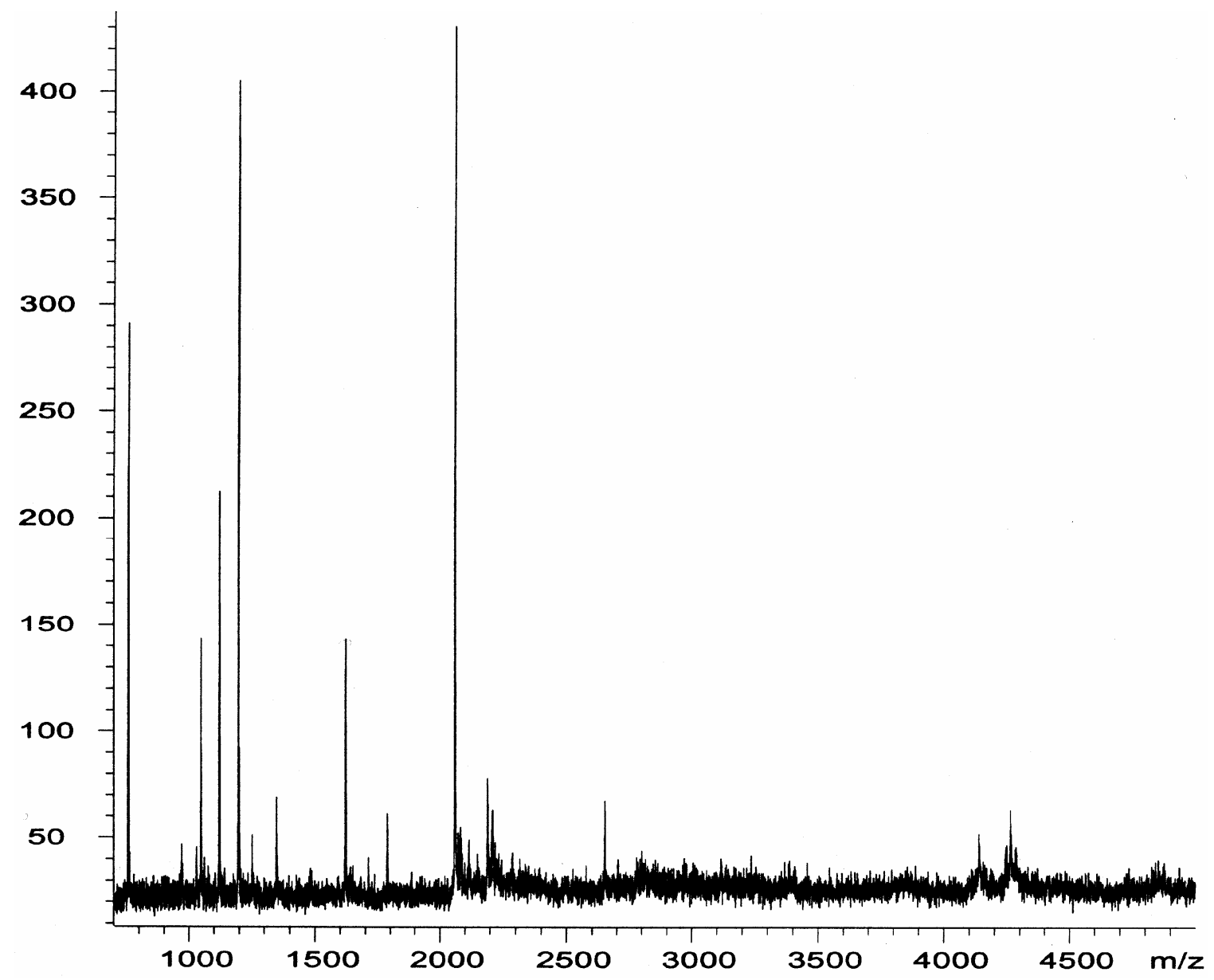

Figure S33. MALDI-TOF mass spectrum of the filtrate after isolation of macrocycle 14, confirming that no larger macrocycles were formed (dithranol matrix). The peaks around $\mathrm{m} / \mathrm{z} 4200$ correspond to dimers of $\mathbf{1 4}$. 